\title{
Hierarchical Archimax copulas
}

\author{
Marius Hofert ${ }^{\mathrm{a}, *}$, Raphaël Huser ${ }^{\mathrm{b}}$, Avinash Prasad ${ }^{\mathrm{a}}$ \\ ${ }^{a}$ Department of Statistics and Actuarial Science, University of Waterloo \\ 200 University Avenue West, Waterloo, ON, Canada N2L 3G1 \\ ${ }^{b}$ Computer, Electrical and Mathematical Science and Engineering Division \\ King Abdullah University of Science and Technology, Saudi Arabia
}

\begin{abstract}
The class of Archimax copulas is generalized to hierarchical Archimax copulas in two ways. First, a hierarchical construction of $d$-norm generators is introduced to construct hierarchical stable tail dependence functions which induce a hierarchical structure on Archimax copulas. Second, by itself or additionally, hierarchical frailties are introduced to extend Archimax copulas to hierarchical Archimax copulas in a similar way as nested Archimedean copulas extend Archimedean copulas. Possible extensions to nested Archimax copulas are discussed. A general formula for the density and its evaluation of Archimax copulas is also introduced.
\end{abstract}

Keywords: Archimax copulas, Hierarchical frailties, Hierarchical stable tail dependence functions, Nesting 2010 MSC: 60E05, 62E15, 62H99

\section{Introduction}

The class of Archimax copulas [5, 7] generalizes Archimedean copulas to incorporate a stable tail dependence function as known from extreme-value copulas. Archimax copulas thus include both Archimedean and extreme-value copulas as special cases. They provide a link between dependence structures arising in multivariate extremes and Archimedean copulas, which have intuitive and computationally appealing properties. One feature of Archimedean copulas is that they can be nested in the sense that under assumptions detailed below, one can plug Archimedean copulas into each other and still obtain a proper copula. Such a construction is hierarchical in the sense that certain multivariate margins are exchangeable, yet the copula overall is not; this additional flexibility to allow for (partial) asymmetry over an exchangeable model is typically used to model components belonging to different groups, clusters or business sectors.

In this work, we raise the following natural question, addressed in Sections 2 and 3: How can hierarchical Archimax copulas be constructed? Since we work with stochastic representations, sampling is also covered. Constructing nested Archimax copulas is largely an open problem which we discuss in Appendix A. Moreover, to fill a gap in the literature, we present a general formula for the density and its evaluation of Archimax copulas; see Appendix B. In what follows, we assume the reader to be familiar with the basics of Archimedean copulas (ACs) and extreme-value copulas (EVCs); see, e.g., [31] for the former (from which we also adopt the notation) and Chapter 6 of [24] for the latter.

\section{Hierarchical extreme-value copulas via hierarchical stable tail dependence functions}

\subsection{Connection between $d$-norms and stable tail dependence functions}

A copula $C$ is an extreme-value copula if and only if it is max-stable, i.e., if $C(\boldsymbol{u})=C^{m}\left(u_{1}^{1 / m}, \ldots, u_{d}^{1 / m}\right)$ for all $m \in \mathbb{N}$ and $\boldsymbol{u}=\left(u_{1}, \ldots, u_{d}\right) \in[0,1]^{d}$; see, e.g., Theorem 6.2.1 in [24]. An extreme-value copula $C$ can

\footnotetext{
* Corresponding author

Email address: marius.hofert@uwaterloo.ca (Avinash Prasad)
} 
be characterized in terms of its stable tail dependence function $\ell:[0, \infty)^{d} \rightarrow[0, \infty)$ via the relation

$$
C(\boldsymbol{u})=\exp \left\{-\ell\left(-\ln u_{1}, \ldots,-\ln u_{d}\right)\right\}
$$

valid for all $\boldsymbol{u} \in[0,1]^{d}$; see, e.g., Section 8.2 in [2] and Chapter 6 in [24]. A characterization of stable tail dependence functions $\ell$ (being homogeneous of order 1 , being 1 when evaluated at the unit vectors in $\mathbb{R}^{d}$ and being fully $d$-max decreasing) is given in [7] and [39].

Sampling from EVCs is often quite challenging and time consuming. Examples which are comparably easy to sample are Gumbel and nested Gumbel copulas, which are the only Archimedean and nested Archimedean EVCs, respectively; in both cases, a stochastic representation is available, as reported in [13] and Theorem 4.5.2 in [34]. Specifically, the Gumbel (or logistic) copula $C$ with parameter $\alpha \in(0,1]$ and stable tail dependence function defined, for all $\boldsymbol{x} \in[0, \infty)^{d}$, by $\ell(\boldsymbol{x})=\left(x_{1}^{1 / \alpha}+\cdots+x_{d}^{1 / \alpha}\right)^{\alpha}$ can be sampled using the algorithm of [28]. It exploits the stochastic representation

$$
\boldsymbol{U}=\left(\psi\left(E_{1} / V\right), \ldots, \psi\left(E_{d} / V\right)\right) \sim C,
$$

where $\psi(t)=e^{-t^{\alpha}}$ is a Gumbel generator, $E_{1}, \ldots, E_{d}$ are mutually independent $\mathcal{E}(1)$, also independent of the frailty $V \sim \mathcal{P} \mathcal{S}(\alpha)=\mathcal{S}\left\{\alpha, 1, \cos ^{1 / \alpha}(\alpha \pi / 2), \mathbf{1}_{(\alpha=1)} ; 1\right\}$; see p. 8 of [36] for the parameterization of this $\alpha$-stable distribution. As for nested Gumbel copulas [42], they can be sampled based on a stochastic representation corresponding to the nesting structure; see [30]. The main idea is to replace the single frailty $V$ by a sequence of dependent frailties (all $\alpha$-stable for different $\alpha$ ), nested in a specific way; see Section 3.

For more complicated EVCs, approximate or exact simulation schemes have been proposed in [9, 10, 40] based on the following stochastic representation of max-stable processes; see [8, 38, 40].

Theorem 1 (Spectral representation of max-stable processes). Let $W_{1}(\boldsymbol{s}), W_{2}(\boldsymbol{s}), \ldots$ be mutually independent copies of the random process $W(s), s \in \mathcal{S} \subseteq \mathbb{R}^{q}$, such that $W(\boldsymbol{s}) \geq 0$ and $\mathrm{E}\{W(\boldsymbol{s})\}=1$ for all $s \in \mathcal{S}$. Furthermore, let $P_{1}, P_{2}, \ldots$ be points of a Poisson point process on $[0, \infty)$ with intensity $x^{-2} \mathrm{~d} x$. Then

$$
Z(s)=\sup _{i \in \mathbb{N}}\left\{P_{i} W_{i}(s)\right\}
$$

is a max-stable random process with unit Fréchet margins and, for all $x_{1}, \ldots, x_{d}>0$,

$$
\ell\left(x_{1}, \ldots, x_{d}\right)=\mathrm{E}\left[\max _{j \in\{1, \ldots, d\}}\left\{x_{j} W\left(\boldsymbol{s}_{j}\right)\right\}\right]
$$

is the associated stable tail dependence function of the random vector $\left(Z\left(s_{1}\right), \ldots, Z\left(s_{d}\right)\right)$ for fixed $\boldsymbol{s}_{1}, \ldots, \boldsymbol{s}_{d}$. Therefore, if a process $Z(s)$ can be expressed as in (3), the distribution function of the random vector $\left(Z\left(\boldsymbol{s}_{1}\right), \ldots, Z\left(\boldsymbol{s}_{d}\right)\right)$ is $\operatorname{Pr}\left\{Z\left(\boldsymbol{s}_{1}\right) \leq x_{1}, \ldots, Z\left(\boldsymbol{s}_{d}\right) \leq x_{d}\right\}=\exp \left\{-\ell\left(1 / x_{1}, \ldots, 1 / x_{d}\right)\right\}$, i.e., $\left(Z\left(\boldsymbol{s}_{1}\right), \ldots, Z\left(\boldsymbol{s}_{d}\right)\right)$ has $E V C C$ with stable tail dependence function $\ell$ and unit Fréchet margins $\exp \left(-1 / x_{j}\right)$ for all $j \in\{1, \ldots, d\}$.

For completeness, Algorithm 1 below describes the traditional approach for simulating max-stable processes constructed using (3). This algorithm goes back to [40] and provides approximate simulations by truncating the supremum to a finite number of processes in (3). When the random process $W(\boldsymbol{s})$ is bounded almost surely, a stopping criterion may be designed to optimally select the number of Poisson points $N$ to perform exact simulation. For more general exact sampling schemes, we refer to [9] and [10].

Algorithm 1. An approximate sampling of max-stable processes based on (3) proceeds as follows:

1. Simulate Poisson points $P_{1}, \ldots, P_{N}$ in decreasing order as $P_{i}=1 /\left(E_{1}+\cdots+E_{i}\right), i \in\{1, \ldots, N\}$, where $E_{1}, \ldots, E_{N} \sim \mathcal{E}(1)$ are mutually independent standard exponential random variables.

2. Simulate $N$ mutually independent copies $W_{1}(s), \ldots, W_{N}(s)$ of the process $W(s)$ at a finite set of locations $s \in\left\{\boldsymbol{s}_{1}, \ldots, \boldsymbol{s}_{d}\right\}$.

3. For each location $s \in\left\{\boldsymbol{s}_{1}, \ldots, \boldsymbol{s}_{d}\right\}$, set $Z(\boldsymbol{s})=\max \left\{P_{1} W_{1}(\boldsymbol{s}), \ldots, P_{N} W_{N}(\boldsymbol{s})\right\}$. 
By choosing the spatial domain $\mathcal{S}$ in $(3)$ to be finite and replacing $W\left(s_{1}\right), \ldots, W\left(s_{d}\right)$ by non-negative random variables $W_{1}, \ldots, W_{d}$ with $\mathrm{E}\left(W_{j}\right)=1$ for all $j \in\{1, \ldots, d\}$, thus replacing the random process $W(s)$ by the non-negative random vector $\boldsymbol{W}=\left(W_{1}, \ldots, W_{d}\right)$, this representation also provides a characterization of, and sampling algorithms for, (finite-dimensional) EVCs; from here on we will adopt this "vector case" for $W$ and accordingly for $Z$.

We now turn to the link between max-stable random vectors $\left(Z_{1}, \ldots, Z_{d}\right)$ and $d$-norms as recently described in [1]. A norm $\|\cdot\|_{d}$ on $\mathbb{R}^{d}$ is called a $d$-norm if there exists a random vector $\boldsymbol{W}=\left(W_{1}, \ldots, W_{d}\right)$ with $W_{j} \geq 0$ and $\mathrm{E}\left(W_{j}\right)=1$ for all $j \in\{1, \ldots, d\}$, such that, for all $\boldsymbol{x}=\left(x_{1}, \ldots, x_{d}\right) \in \mathbb{R}^{d}$,

$$
\|\boldsymbol{x}\|_{d}=\mathrm{E}\left\{\max \left(\left|x_{1}\right| W_{1}, \ldots,\left|x_{d}\right| W_{d}\right)\right\}=\mathrm{E}\left(\|\boldsymbol{x} \boldsymbol{W}\|_{\infty}\right)
$$

where $\|\cdot\|_{\infty}$ denotes the supremum norm and $\boldsymbol{x} \boldsymbol{W}$ is understood componentwise. In this case, $\boldsymbol{W}$ is called generator of $\|\cdot\|_{d}$. One can compare (4) and (5) to identify the correspondence, valid for all $\boldsymbol{x} \in[0, \infty)^{d}$,

$$
\ell(\boldsymbol{x})=\|\boldsymbol{x}\|_{d}=\mathrm{E}\left(\|\boldsymbol{x} \boldsymbol{W}\|_{\infty}\right)
$$

between $d$-norms and stable tail dependence functions on $[0, \infty)^{d}$. Specifying a generator $\boldsymbol{W}$ thus defines a stable tail dependence function which in turn characterizes an EVC. The link (6) with $d$-norms provides us with a useful method for constructing and sampling EVCs which can also be exploited for constructing hierarchical EVCs (HEVCs).

We now provide a few examples of $d$-norm generators for well known copulas which can serve as building blocks for HEVCs (and, see Section 3, hierarchical Archimax copulas).

Example 1. a) If $\boldsymbol{W}=(1, \ldots, 1)$ with probability one, then $\|\boldsymbol{x}\|_{d}=\max \left(\left|x_{1}\right|, \ldots,\left|x_{d}\right|\right)$. This characterizes comonotonicity, i.e., the upper Fréchet-Hoeffding bound with stable tail dependence function $\ell(\boldsymbol{x})=\max \left(x_{1}, \ldots, x_{d}\right)$.

b) If $\boldsymbol{W}$ is a random permutation of $(d, 0, \ldots, 0) \in \mathbb{R}^{d}$, then $\|\boldsymbol{x}\|_{d}=\left|x_{1}\right|+\cdots+\left|x_{d}\right|$. This characterizes independence with the stable dependence function $\ell(\boldsymbol{x})=x_{1}+\cdots+x_{d}$.

c) If $\boldsymbol{W}=\left(W_{1}, \ldots, W_{d}\right)$ is such that for some $\alpha \in(0,1), \Gamma(1-\alpha) W_{j} \stackrel{\text { ind. }}{\sim} e^{-x^{-1 / \alpha}}, x \in[0, \infty)$, where $\Gamma$ denotes Euler's Gamma function, a straightforward computation shows that

$$
\|\boldsymbol{x}\|_{d}=\left(\left|x_{1}\right|^{1 / \alpha}+\cdots+\left|x_{d}\right|^{1 / \alpha}\right)^{\alpha}, \quad \ell(\boldsymbol{x})=\left(x_{1}^{1 / \alpha}+\cdots+x_{d}^{1 / \alpha}\right)^{\alpha}
$$

and thus the max-stable dependence structure is the Gumbel (logistic) copula with parameter $\alpha \in(0,1)$.

d) If $\boldsymbol{W}$ is such that for some $\theta>0, W_{j}=\Gamma(1+1 / \theta) W_{j}^{*}$ with $W_{j}^{*} \stackrel{\text { ind. }}{\sim} \exp \left(-x^{\theta}\right), x \in[0, \infty)$, then the stable tail dependence function can be calculated to be

$$
\ell(\boldsymbol{x})=\sum_{\emptyset \neq J \subseteq\{1, \ldots, d\}}(-1)^{|J|+1}\left(\sum_{j \in J} x_{j}^{-\theta}\right)^{-1 / \theta},
$$

and thus the max-stable dependence structure is the negative logistic copula with parameter $\theta>0$; see, e.g., [10].

e) If $\boldsymbol{W}=\left(W_{1}, \ldots, W_{d}\right) \sim\left(\sqrt{2 \pi} \max \left(0, \varepsilon_{1}\right), \ldots, \sqrt{2 \pi} \max \left(0, \varepsilon_{d}\right)\right)$, where $\left(\varepsilon_{1}, \ldots, \varepsilon_{d}\right) \sim \mathcal{N}_{d}(\mathbf{0}, P)$ with correlation matrix $P$, a Schlather model results; see [40].

f) If $\boldsymbol{W}=\left(W_{1}, \ldots, W_{d}\right) \sim\left(\max \left(0, \varepsilon_{1}\right)^{\nu} / c_{\nu}, \ldots, \max \left(0, \varepsilon_{d}\right)^{\nu} / c_{\nu}\right)$, where $\left(\varepsilon_{1}, \ldots, \varepsilon_{d}\right) \sim \mathcal{N}_{d}(\mathbf{0}, P)$ with correlation matrix $P, \nu>0, c_{\nu}=2^{\nu / 2-1} \Gamma\{(\nu+1) / 2\} / \sqrt{\pi}$, then the extremal Student $t$ model of 
[37] results; for $\nu=1$, the Schlather model is obtained as a special case. The stable tail dependence function $\ell(\boldsymbol{x})$ of the extremal $t$ model in dimension $d$ is given by

$$
\ell(\boldsymbol{x})=\sum_{j=1}^{d} x_{j} t_{d-1}\left(\nu+1, P_{-j, j}, \frac{P_{-j,-j}-P_{-j, j} P_{j,-j}}{\nu+1}\right)\left\{\left(\boldsymbol{x}_{-j} / x_{j}\right)^{-1 / \nu}\right\},
$$

where $t_{d}(\nu, \boldsymbol{\mu}, \Sigma)(\boldsymbol{x})$ denotes the $d$-variate Student $t$ distribution function with $\nu$ degrees of freedom, location vector $\boldsymbol{\mu}$ and dispersion matrix $\Sigma$ evaluated at $\boldsymbol{x}$ as in Example 6.7 of [32], $P_{-j,-j}$ (respectively, $P_{-j, j}, P_{j,-j}$ ) denotes the submatrix obtained by removing the $j$ th row and the $j$ th column (respectively, $j$ th row, $j$ th column) from $P$ and $\boldsymbol{x}_{-j}=\left(x_{1}, \ldots, x_{j-1}, x_{j+1}, \ldots, x_{d}\right)$.

g) If $\boldsymbol{W}=\left(W_{1}, \ldots, W_{d}\right) \sim\left(\exp \left(\varepsilon_{1}-\sigma_{1}^{2} / 2\right), \ldots, \exp \left(\varepsilon_{d}-\sigma_{d}^{2} / 2\right)\right)$, where $\left(\varepsilon_{1}, \ldots, \varepsilon_{d}\right) \sim \mathcal{N}_{d}(\mathbf{0}, \Sigma)$ for a covariance matrix $\Sigma$ with diagonal entries $\Sigma_{j j}=\sigma_{j}^{2}, j \in\{1, \ldots, d\}$, corresponding correlation matrix $P$ (such that $\Sigma_{i j}=\sigma_{i} \sigma_{j} P_{i j}, i, j \in\{1, \ldots, d\}$ ), a Brown-Resnick model results; see [26]. This model can also be obtained as a certain limit of the extremal $t$ model when the degrees of freedom $\nu \rightarrow \infty$; see [35]. The Brown-Resnick model is characterized by the Hüsler-Reiß copula; see [23]. Its stable tail dependence function $\ell(\boldsymbol{x})$ is available in any dimension $d$, see [22] and [35], given by

$$
\ell(\boldsymbol{x})=\sum_{j=1}^{d} x_{j} \Phi_{d-1}\left(\mathbf{0}, \Sigma_{j}\right)\left(\boldsymbol{\eta}_{j}\right)
$$

where $\Phi_{d}(\boldsymbol{\mu}, \Sigma)(\boldsymbol{x})$ denotes the $d$-variate normal distribution function with mean vector $\boldsymbol{\mu}$ and covariance matrix $\Sigma$ evaluated at $\boldsymbol{x}, \Sigma_{j}$ is the $(d-1) \times(d-1)$ covariance matrix with entries

$$
\Sigma_{j, i k}= \begin{cases}2 \gamma_{i j}, & \text { if } k=i \in\{1, \ldots, d\} \backslash\{j\} \\ \gamma_{i j}+\gamma_{j k}-\gamma_{i k}, & \text { if } k \neq i\end{cases}
$$

where $\gamma_{i j}=\sigma_{i}^{2}+\sigma_{j}^{2}-\sigma_{i} \sigma_{j} P_{i j}, \boldsymbol{\eta}_{j}$ is the $(d-1)$-dimensional vector with $i$ th entry $\gamma_{i j}-\ln \left(x_{i} / x_{j}\right)$.

h) If $\boldsymbol{W}=\left(W_{1}, \ldots, W_{d}\right) \sim H$ for a distribution function $H$ with margins $F_{1}, \ldots, F_{d}$ on $[0, \infty)$ such that $\mathrm{E}\left(W_{j}\right)=1$ for all $j \in\{1, \ldots, d\}$, then, by Sklar's Theorem, if $C$ denotes the copula of $H$, one can derive the general form of $\ell$ via (6). If $\boldsymbol{U} \sim C$, then the stochastic representation $\boldsymbol{W}=\left(F_{1}^{-}\left(U_{1}\right), \ldots, F_{d}^{-}\left(U_{d}\right)\right)$ can be used to see that, for all $\boldsymbol{x}>\mathbf{0}$,

$$
\begin{aligned}
G_{\boldsymbol{x}}(y) & =\operatorname{Pr}\left\{\max \left(\left|x_{1}\right| W_{1}, \ldots,\left|x_{d}\right| W_{d}\right) \leq y\right\}=\operatorname{Pr}\left(W_{1} \leq y / x_{1}, \ldots, W_{d} \leq y / x_{d}\right) \\
& =\operatorname{Pr}\left\{U_{1} \leq F_{1}\left(y / x_{1}\right), \ldots, U_{d} \leq F_{d}\left(y / x_{d}\right)\right\}=C\left\{F_{1}\left(y / x_{1}\right), \ldots, F_{d}\left(y / x_{d}\right)\right\} .
\end{aligned}
$$

Applying the chain rule for differentiating this expression with respect to $y$ leads to the density

$$
g_{\boldsymbol{x}}(y)=\sum_{j=1}^{d} \mathrm{D}_{j} C\left\{F_{1}\left(y / x_{1}\right), \ldots, F_{d}\left(y / x_{d}\right)\right\} f_{j}\left(y / x_{j}\right) / x_{j},
$$

where $\mathrm{D}_{j} C(\boldsymbol{u})$ denotes the partial derivatives of $C$ with respect to the $j$ th argument evaluated at $\boldsymbol{u}$. By (6) and the substitution $z_{j}=y / x_{j}$, we thus have that, for all $\boldsymbol{x}>\mathbf{0}$,

$$
\begin{aligned}
\ell(\boldsymbol{x}) & =\int_{0}^{\infty} y g_{\boldsymbol{x}}(y) \mathrm{d} y=\sum_{j=1}^{d} \frac{1}{x_{j}} \int_{0}^{\infty} y \mathrm{D}_{j} C\left\{F_{1}\left(y / x_{1}\right), \ldots, F_{d}\left(y / x_{d}\right)\right\} f_{j}\left(y / x_{j}\right) \mathrm{d} y \\
& =\sum_{j=1}^{d} x_{j} \int_{0}^{\infty} z_{j} \mathrm{D}_{j} C\left\{F_{1}\left(z_{j} x_{j} / x_{1}\right), \ldots, F_{d}\left(z_{j} x_{j} / x_{d}\right)\right\} f_{j}\left(z_{j}\right) \mathrm{d} z_{j} \\
& =\sum_{j=1}^{d} x_{j} \mathrm{E}\left[Z_{j} \mathrm{D}_{j} C\left\{F_{1}\left(Z_{j} x_{j} / x_{1}\right), \ldots, F_{d}\left(Z_{j} x_{j} / x_{d}\right)\right\}\right],
\end{aligned}
$$


where $Z_{1} \sim F_{1}, \ldots, Z_{d} \sim F_{d}$ are independent. This formula resembles (7) and (8). If required, it can be evaluated by Monte Carlo, for example. Note that it only poses a restriction on the marginal distributions (being non-negative and scalable to have mean 1), not the dependence of the components of $\boldsymbol{W}$.

\subsection{Hierarchical stable tail dependence functions}

Let us now turn to a construction method for HEVCs by exploiting the link between $d$-norm generators and stable tail dependence functions established in Section 2.1. The idea is to build stable tail dependence functions with a hierarchical structure on the level of the associated $d$-norm generator. Although our approach is similar in spirit to [27], where factor extreme-value copula models are proposed, the two constructions differ.

By analogy with the construction of nested Archimedean copulas (outlined in Section 3) we define hierarchical $d$-norm generators $\boldsymbol{W}=\left(W_{1}, \ldots, W_{d}\right)$ in terms of a tree structure with $d$ leaves. Under this framework, each component $W_{j}$, with $j \in\{1, \ldots, d\}$, is obtained as a measurable, non-negative function $g_{j}$ of intermediate variables $\left\{W_{k}^{*}: k \in \operatorname{Anc}(j)\right\}$, lying on the tree nodes along the path from the seed $W_{0}^{*}$ at the root of the tree to the $j$ th leaf represented by the variable $W_{j}$ itself. In other words, the variable $W_{j}$ may be expressed in terms of its ancestor variables identified by the index set $\operatorname{Anc}(j)$, some of which may be shared with other variables $W_{k}, k \neq j$, thus inducing dependence between the components of the vector $\boldsymbol{W}$.

To fix ideas, consider the tree represented in Figure 1. In this case, one has, for example, $W_{2}=$ $g_{2}\left(W_{0}^{*}, W_{2}^{*}, W_{21}^{*}\right)$ and $W_{7}=g_{7}\left(W_{0}^{*}, W_{3}^{*}, W_{32}^{*}, W_{323}^{*}\right)$. To define a valid $d$-norm generator, we need to assume that this system of variables and the corresponding functions $g_{j}$ are such that $\mathrm{E}\left(W_{j}\right)=1$ for each $j \in\{1, \ldots, d\}$. However, there is no further restriction on the dependence structure of these latent variables, which yields a very general framework.

The inherent hierarchical structure of the $d$-norm generator defined in this way carries over to the EVC derived from (4). Such hierarchical $d$-norm generators yield HEVCs.

We now describe several example models of HEVCs constructed using this general framework. We first consider the well-known nested Gumbel copula and show that it arises as HEVCs in our framework; see $[29,41,42]$ for early references. Nested Gumbel (or logistic) copulas have been applied in a variety of applications, such as [18] in the realm of pricing collateralized debt obligations or [43] where they are used to group various air pollutants into clusters with homogeneous extremal dependence strength.

Example 2 (Nested Gumbel copulas with two nesting levels). For $0<\alpha_{1}, \ldots, \alpha_{S} \leq \alpha_{0} \leq 1$, consider independent random variables organized in $S$ groups:

Root: $W_{0}^{*}=1$,

Level 1: $\quad W_{s}^{*} \stackrel{\text { ind. }}{\sim} \mathcal{P} \mathcal{S}\left(\alpha_{s} / \alpha_{0}\right), \quad s \in\{1, \ldots, S\}$,

Level 2: $W_{s j}^{*} \stackrel{\text { ind. }}{\sim} \exp \left(-x^{-1 / \alpha_{s}}\right), x>0, \quad s \in\{1, \ldots, S\}, j \in\left\{1, \ldots, d_{s}\right\}$.

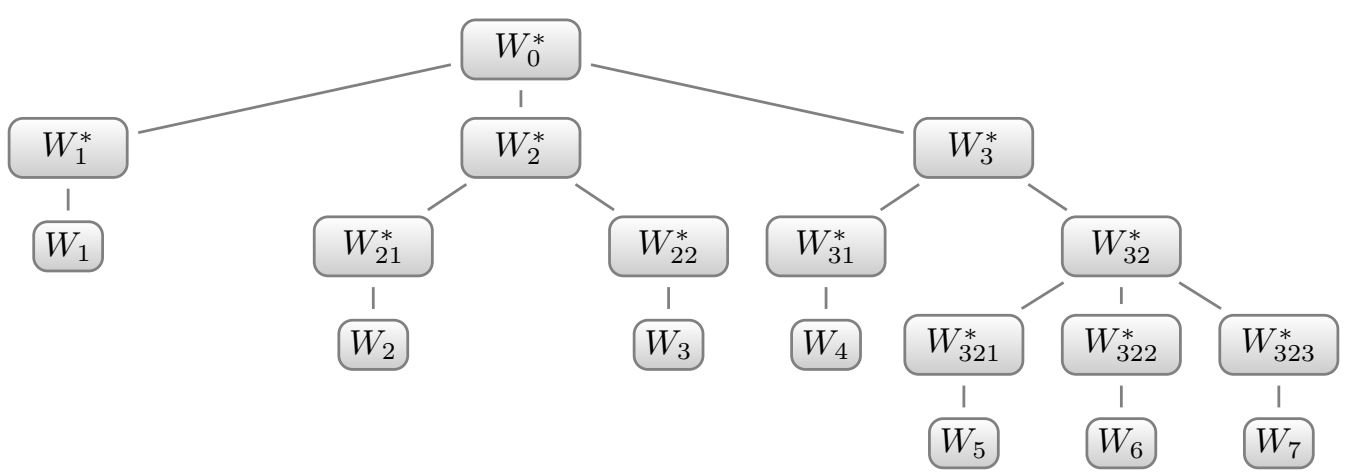

Figure 1: Tree representation of a hierarchical $d$-norm generator with $d=7$ for the construction of a HEVC. 
As outlined above, the leaves of the tree correspond to the $d$-norm generator $\boldsymbol{W}=\left(\boldsymbol{W}_{1}, \ldots, \boldsymbol{W}_{S}\right)$, with $\boldsymbol{W}_{s}=\left(W_{s 1}, \ldots, W_{s d_{s}}\right), s \in\{1, \ldots, S\}$, with $d=d_{1}+\cdots+d_{S}$, where, for all $s \in\{1, \ldots, S\}$ and $j \in\left\{1, \ldots, d_{s}\right\}$,

$$
W_{s j}=g_{s j}\left(W_{0}^{*}, W_{s}^{*}, W_{s j}^{*}\right)=\frac{W_{s}^{* \alpha_{s}} W_{s j}^{*}}{\Gamma\left(1-\alpha_{0}\right)} .
$$

It can be verified that, indeed, $W_{s j} \geq 0$ and $\mathrm{E}\left(W_{s j}\right)=1$ for all $s$ and $j$. Then, the stable tail dependence function corresponding to the $d$-norm generator $\boldsymbol{W}$ is given by

$$
\ell(\boldsymbol{x})=\ell_{\alpha_{0}}\left\{\ell_{\alpha_{1}}\left(\boldsymbol{x}_{1}\right), \ldots, \ell_{\alpha_{S}}\left(\boldsymbol{x}_{S}\right)\right\}
$$

where $\boldsymbol{x}=\left(\boldsymbol{x}_{1}, \ldots, \boldsymbol{x}_{S}\right), \boldsymbol{x}_{s}=\left(x_{s 1}, \ldots, x_{s d_{s}}\right), s \in\{1, \ldots, S\}, \ell_{\alpha}\left(x_{1}, \ldots, x_{d}\right)=\left(x_{1}^{1 / \alpha}+\cdots+x_{d}^{1 / \alpha}\right)^{\alpha}$ is the stable tail dependence function of a Gumbel copula with parameter $\alpha$.

Proof. It directly follows from (6) that, for all $\boldsymbol{x} \in[0, \infty)^{d}$,

$$
\ell(\boldsymbol{x})=\mathrm{E}\left[\max _{s \in\{1, \ldots, S\}}\left\{\max _{j \in\left\{1, \ldots d_{s}\right\}}\left(x_{s j} W_{s j}\right)\right\}\right] .
$$

By (9) and with $Y_{\boldsymbol{x}}=\max _{s \in\{1, \ldots, S\}}\left\{\max _{j \in\left\{1, \ldots, d_{s}\right\}}\left(x_{s j} W_{s}^{* \alpha_{s}} W_{s j}^{*}\right)\right\}$, we get that, for all $\boldsymbol{x} \in[0, \infty)^{d}$,

$$
\begin{aligned}
\ell(\boldsymbol{x}) & =\frac{1}{\Gamma\left(1-\alpha_{0}\right)} \mathrm{E}\left[\max _{s \in\{1, \ldots, S\}}\left\{\max _{j \in\left\{1, \ldots, d_{s}\right\}}\left(x_{s j} W_{s}^{* \alpha_{s}} W_{s j}^{*}\right)\right\}\right]=\frac{1}{\Gamma\left(1-\alpha_{0}\right)} \mathrm{E}\left(Y_{\boldsymbol{x}}\right) \\
& =\frac{1}{\Gamma\left(1-\alpha_{0}\right)} \int_{0}^{\infty} \operatorname{Pr}\left(Y_{\boldsymbol{x}}>y\right) \mathrm{d} y .
\end{aligned}
$$

Conditioning on $W_{s}^{*}, s \in\{1, \ldots, S\}$, we find that

$$
\begin{aligned}
\operatorname{Pr}\left(Y_{\boldsymbol{x}} \leq y\right) & =\operatorname{Pr}\left\{W_{s j}^{*} \leq y /\left(x_{s j} W_{s}^{* \alpha_{s}}\right), s \in\{1, \ldots, S\}, j \in\left\{1, \ldots, d_{s}\right\}\right\} \\
& =\mathrm{E}\left[\prod_{s=1}^{S} \prod_{j=1}^{d_{s}} \exp \left\{\left(-\frac{y}{x_{s j} W_{s}^{* \alpha_{s}}}\right)^{-1 / \alpha_{s}}\right\}\right]=\prod_{s=1}^{S} \mathrm{E}\left[\exp \left\{-W_{s}^{*} \sum_{j=1}^{d_{s}}\left(\frac{y}{x_{s j}}\right)^{-1 / \alpha_{s}}\right\}\right],
\end{aligned}
$$

where the last equality holding since $W_{1}^{*}, \ldots, W_{S}^{*}$ are independent. Since $W_{s}^{*} \sim \mathcal{P} \mathcal{S}\left(\alpha_{s} / \alpha_{0}\right)$, this leads to

$$
\operatorname{Pr}\left(Y_{\boldsymbol{x}} \leq y\right)=\prod_{s=1}^{S} \exp \left[-\left\{\sum_{j=1}^{d_{s}}\left(y / x_{s j}\right)^{-1 / \alpha_{s}}\right\}^{\alpha_{s}} / \alpha_{0}\right]=\exp \left[-y^{-1 / \alpha_{0}}\left[\sum_{s=1}^{S}\left\{\sum_{j=1}^{d_{s}}\left(x_{s j}^{1 / \alpha_{s}}\right)\right\}^{\alpha_{s} / \alpha_{0}}\right]\right]
$$

With

$$
t=\sum_{s=1}^{S}\left\{\sum_{j=1}^{d_{s}}\left(x_{s j}^{1 / \alpha_{s}}\right)\right\}^{\alpha_{s} / \alpha_{0}}
$$

the substitution $z=y^{-1 / \alpha_{0}} t$, integration by parts, the stable tail dependence function is thus

$$
\begin{aligned}
\ell(\boldsymbol{x}) & =\frac{1}{\Gamma\left(1-\alpha_{0}\right)} \int_{0}^{\infty}\left\{1-\exp \left(-y^{-1 / \alpha_{0}} t\right)\right\} \mathrm{d} y=\frac{t^{\alpha_{0}}}{\Gamma\left(1-\alpha_{0}\right)} \int_{0}^{\infty}\{1-\exp (-z)\} \alpha_{0} z^{-\alpha_{0}-1} \mathrm{~d} z \\
& =\frac{t^{\alpha_{0}}}{\Gamma\left(1-\alpha_{0}\right)} \int_{0}^{\infty} z^{-\alpha_{0}} \exp (-z) \mathrm{d} z=\frac{t^{\alpha_{0}}}{\Gamma\left(1-\alpha_{0}\right)} \Gamma\left(1-\alpha_{0}\right)=t^{\alpha_{0}}=\left\{\sum_{s=1}^{S}\left(\sum_{j=1}^{d_{s}} x_{s j}^{1 / \alpha_{s}}\right)^{\alpha_{s} / \alpha_{0}}\right\}^{\alpha_{0}} \\
& =\ell_{\alpha_{0}}\left\{\ell_{\alpha_{1}}\left(\boldsymbol{x}_{1}\right), \ldots, \ell_{\alpha_{S}}\left(\boldsymbol{x}_{S}\right)\right\}
\end{aligned}
$$

which is the stable tail dependence function of a nested Gumbel copula constructed by nesting on the level of the $d$-norms. 
The construction underlying Example 2 may easily be generalized to trees with arbitrary nesting levels using the same line of proof. The construction, extending [41], is outlined in Example 3.

Example 3 (Nested Gumbel copulas with arbitrary nesting levels). To construct a nested Gumbel copula with arbitrary nesting levels, we mimic the construction with two nesting levels in Example 2. Let $p_{j}$ be the path starting from the root of the tree and leading to the $j$ th leaf representing the $d$-norm generator component $W_{j}$. We can write the corresponding node variables along this path as $W_{0}^{*}, W_{p_{j}(1)}^{*}, W_{p_{j}(2)}^{*}, \ldots, W_{p_{j}\left(L_{j}\right)}^{*}, W_{j}$, where $L_{j}$ denotes the number of intermediate variables (or levels) between $W_{0}^{*}$ and $W_{j}$. Assume that all latent variables $W_{p_{j}(k)}^{*}$ with $j \in\{1, \ldots, d\}$ and $k \in\left\{1, \ldots, L_{j}\right\}$, are mutually independent within and across paths, that

$$
\begin{aligned}
& \text { Root: } \quad W_{0}^{*}=1 \text {, } \\
& \text { Level 1: } \quad W_{p_{j}(1)}^{*} \sim \mathcal{P} \mathcal{S}\left(\alpha_{p_{j}(1)} / \alpha_{0}\right), \\
& \text { Level } k: \quad W_{p_{j}(k)}^{*} \sim \mathcal{P} \mathcal{S}\left(\alpha_{p_{j}(k)} / \alpha_{p_{j}(k-1)}\right), \quad k \in\left\{2, \ldots, L_{j}-1\right\} \text {, } \\
& \text { Level } L_{j}: \quad W_{p_{j}\left(L_{j}\right)}^{*} \sim \exp \left(-x^{\left.-1 / \alpha_{p_{j}\left(L_{j}-1\right)}\right)}, \quad x>0,\right.
\end{aligned}
$$

where, for each path $p_{j}$, the parameters of the positive $\alpha$-stable variables on this path are ordered as $0<\alpha_{p_{j}\left(L_{j}-1\right)} \leq \cdots \leq \alpha_{p_{j}(1)} \leq \alpha_{0}<1$. For each $j \in\{1, \ldots, d\}$, we can then construct the component $W_{j}$ of the $d$-norm generator via

$$
W_{j}=g_{j}\left(W_{0}^{*}, W_{p_{j}(1)}^{*}, \ldots, W_{p_{j}\left(L_{j}\right)}^{*}\right)=\frac{W_{p_{j}(1)}^{* \alpha_{p_{j}(1)}} \cdots W_{p_{j}\left(L_{j}-1\right)}^{* \alpha_{p_{j}\left(L_{j}-1\right)}} W_{p_{j}\left(L_{j}\right)}^{*}}{\Gamma\left(1-\alpha_{0}\right)} .
$$

By recursively conditioning on the variables along each path, one can show that the resulting $d$-norm generator corresponds to the nested Gumbel copula based on the same tree structure and that its stable tail dependence function can be obtained by applying (10) recursively at each nesting level of the tree.

The construction principle for hierarchical $d$-norm generators also allows us to construct the following two HEVCs.

Example 4 (Hierarchical Hüsler-Reiß copula). For simplicity, consider the two-level case

$$
\begin{array}{rlrl}
\text { Root: } & & W_{0}^{*} & =1, \\
\text { Level 1: } & \left(W_{1}^{*}, \ldots, W_{S}^{*}\right) & \sim \mathcal{N}_{S}\left(\mathbf{0}, \Sigma_{0}\right), \\
\text { Level 2: } & \left(W_{s 1}^{*}, \ldots, W_{s d_{s}}^{*}\right) & \sim \mathcal{N}_{d_{s}}\left(\mathbf{0}, \Sigma_{s}\right), \quad s \in\{1, \ldots, S\},
\end{array}
$$

where the vectors $\left(W_{1}^{*}, \ldots, W_{S}^{*}\right)$ and $\left(W_{s 1}^{*}, \ldots, W_{s d_{s}}^{*}\right)$ with $s \in\{1, \ldots, S\}$, are independent. Furthermore, assume that the covariance matrix $\Sigma_{0}$ may be expressed in terms of the variances $\sigma_{1}^{* 2}, \ldots, \sigma_{S}^{* 2}$ and the correlation matrix $P_{0}$ via $\Sigma_{0, i k}=\operatorname{cov}\left(W_{i}^{*}, W_{k}^{*}\right)=\sigma_{i}^{*} \sigma_{k}^{*} P_{0, i k}$. Similarly, denote by $\sigma_{s 1}^{* 2}, \ldots, \sigma_{s d_{s}}^{* 2}$ and $P_{s}$ the respective quantities for the vector $\left(W_{s 1}^{*}, \ldots, W_{s d_{s}}^{*}\right)$ with $s \in\{1, \ldots, S\}$. Writing the $d$-norm generator as $\boldsymbol{W}=\left(\boldsymbol{W}_{1}, \ldots, \boldsymbol{W}_{S}\right)$, with $\boldsymbol{W}_{s}=\left(W_{s 1}, \ldots, W_{s d_{s}}\right), s \in\{1, \ldots, S\}$, as in Example 2, we define the components by

$$
W_{s j}=\exp \left\{\left(W_{s}^{*}+W_{s j}^{*}\right)-\left(\sigma_{s}^{* 2}+\sigma_{s j}^{* 2}\right) / 2\right\}
$$

for $s \in\{1, \ldots, S\}$ and $j \in\left\{1, \ldots, d_{s}\right\}$. It is immediate from Part 1 of Example 1 and by writing $\varepsilon_{s j}=W_{s}^{*}+W_{s j}^{*}$ that the resulting extreme-value distribution has the Hüsler-Reiß copula as underlying dependence structure. It is characterized by an overall dispersion matrix $\Sigma$ whose entries are given by

$$
\operatorname{cov}\left(\varepsilon_{s_{1} j_{1}}, \varepsilon_{s_{2} j_{2}}\right)= \begin{cases}\Sigma_{0, s_{1} s_{1}}+\Sigma_{s_{1}, j_{1} j_{2}}=\sigma_{s_{1}}^{* 2}+\sigma_{s_{1} j_{1}}^{*} \sigma_{s_{1} j_{2}}^{*} P_{s_{1}, j_{1} j_{2}}, & s_{1}=s_{2} \text { (same groups), } \\ \Sigma_{0, s_{1} s_{2}}=\sigma_{s_{1}}^{*} \sigma_{s_{2}}^{*} P_{0, s_{1} s_{2}}, & s_{1} \neq s_{2} \text { (different groups). }\end{cases}
$$


Hence, in this case, the underlying hierarchical $d$-norm generator results in a hierarchical structure of the covariance matrix $\Sigma$ and the corresponding stable tail dependence function is of the same form. It is straightforward to verify that this hierarchical structure allows to model stronger dependence within groups than between groups. This simple two-level example can easily be generalized to trees with arbitrary nesting levels, it could be interesting for spatial modeling, where different homogeneous regions exhibit different extreme-value behaviors.

Example 5 (Hierarchical extremal $t$ and Schlather copula). Example 4 can be adapted to a hierarchical extremal $t$ model by replacing $(11)$ by $W_{s j}=\max \left\{0,\left(W_{s}^{*}+W_{s j}^{*}\right) /\left(\sigma_{s}^{* 2}+\sigma_{s j}^{* 2}\right)^{1 / 2}\right\}^{\nu} / c_{\nu}$ for all $s \in\{1, \ldots, S\}$ and $j \in\left\{1, \ldots, d_{s}\right\}$, where $\nu>0$ is the degree of freedom and $c_{\nu}$ is the same constant appearing in Part 1 of Example 1. For $\nu=1$, we obtain a hierarchical Schlather model.

\section{Hierarchical Archimax copulas}

\subsection{Archimax copulas}

Let $\Psi$ be the set of all (Archimedean) generators, i.e., all $\psi:[0, \infty) \rightarrow[0,1]$ which are continuous, decreasing, strictly decreasing on $[0, \inf \{t: \psi(t)=0\}]$ and satisfy $\psi(0)=1$ and $\psi(\infty)=\lim _{t \rightarrow \infty} \psi(t)=0$. According to [5] and [7], a copula is an Archimax copula (AXC) if it can be expressed in the form

$$
C(\boldsymbol{u})=\psi\left[\ell\left\{\psi^{-1}\left(u_{1}\right), \ldots, \psi^{-1}\left(u_{d}\right)\right\}\right],
$$

for all $\boldsymbol{u} \in[0,1]^{d}$ for an Archimedean generator $\psi \in \Psi$ and a stable tail dependence function $\ell$; note that the form (12) in $d$ dimensions was originally conjectured in [33]. In what follows, we focus on the case where $\psi$ is completely monotone. Since $\psi(0)=1$, Bernstein's Theorem, see [4] or p. 439 of [12], implies that $\psi$ is the Laplace-Stieltjes transform of a distribution function $F$ on the positive real line, i.e., $\psi(t)=\mathcal{L} \mathcal{S}[F](t)=\int_{0}^{\infty} \exp (-t x) \mathrm{d} F(x), t \in[0, \infty)$, in this case. A stochastic representation for $\boldsymbol{U} \sim C$ is given by

$$
\boldsymbol{U}=\left(\psi\left(E_{1} / V\right), \ldots, \psi\left(E_{d} / V\right)\right)=\left(\psi\left\{-\ln \left(Y_{1}\right) / V\right\}, \ldots, \psi\left\{-\ln \left(Y_{d}\right) / V\right\}\right) \sim C,
$$

where $\left(E_{1}, \ldots, E_{d}\right)=\left(-\ln Y_{1}, \ldots,-\ln Y_{d}\right)$ (which has $\mathcal{E}(1)$ margins) for $\boldsymbol{Y}=\left(Y_{1}, \ldots, Y_{d}\right) \sim D$ for a $d$-dimensional EVC $D$ with stable tail dependence function $\ell$ and $V \sim F=\mathcal{L S}^{-1}[\psi]$ is the frailty in the construction (which is independent of $\boldsymbol{Y}$ ). Note that, as a special case, if $D$ is the independence copula, in other words $\ell(\boldsymbol{x})=x_{1}+\cdots+x_{d}$, then $C$ in (12) is Archimedean. Moreover, if $\psi(t)=e^{-t}, t \geq 0$, then $C$ in (12) is an EVC with stable tail dependence function $\ell$ (compare with (1)) and $\boldsymbol{U}=\boldsymbol{Y}$, so $C=D$. Although not relevant for the remainder of this paper, but important for statistical applications, let us mention that, if it exists, the density of an AXC allows for a rather explicit form (derived in Proposition 3) which makes computing the logarithmic density numerically feasible (see Proposition 4).

\subsection{Two ways of inducing hierarchies}

There are two immediate ways to introduce a hierarchical structure on Archimax copulas following from (13), thus leading to hierarchical Archimax copulas (HAXCs): At the level of the EVC $D$ through its stable tail dependence function (via $d$-norms) and at the level of the frailty $V$ by using a sequence of dependent frailties instead of a single $V$. Since the former was addressed in Section 2, we now focus on the latter.

Let $D$ be a $d$-dimensional EVC with stable tail dependence function $\ell$ as before. The stochastic representation (13) can be generalized by replacing the single frailty $V$ by a sequence of dependent frailties. Their hierarchical structure and dependence is best described in terms of a concrete example. To this end, consider Figure 2.

The hierarchical frailties are shown as nodes and the corresponding (dependent) $\mathcal{E}(1)$ random variables as leaves. The frailty at each level is drawn from a distribution on the positive real line which depends on the frailty from one level before: First $V_{0} \sim F_{0}$ is drawn; then, independently of each other, $V_{01} \sim F_{01}\left(\cdot ; V_{0}\right)$ and $V_{02} \sim F_{02}\left(\cdot ; V_{0}\right)$ are drawn (note that $V_{0}$ thus acts as a parameter on the distributions $F_{01}$ of $V_{01}$ and 


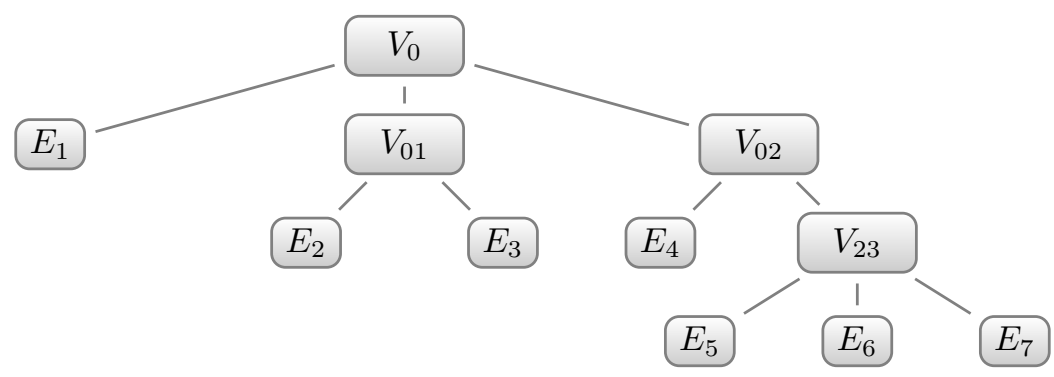

Figure 2: Tree representation of hierarchical frailties for the construction of a HAXC.

$F_{02}$ of $\left.V_{02}\right)$; finally, $V_{23} \sim F_{23}\left(\cdot ; V_{02}\right)$ is drawn. This procedure can easily be generalized (level by level) to more hierarchical levels if so desired. Similar to the Archimax case, if $\left(E_{1}, \ldots, E_{7}\right)$ has EVC $D$ and $\mathcal{E}(1)$ margins, one considers

$$
\left(E_{1} / V_{0}, E_{2} V_{01}, E_{3} / V_{01}, E_{4} / V_{02}, E_{5} / V_{23}, E_{6} / V_{23}, E_{7} / V_{23}\right)
$$

and the survival copula of this random vector is then the HAXC $C$. For the latter step one needs the marginal survival functions of this random vector which are typically not known explicitly. However, they are known under the so-called sufficient nesting condition which is based on certain Laplace-Stieltjes transforms involved and which is also utilized in the construction of nested Archimedean copulas (NACs); see, e.g., $[16,30]$ or pp. 87 of $[25]$.

To introduce these Laplace-Stieltjes transforms, it is convenient to have the construction principle of NACs in mind. The NAC corresponding to Figure 2 is given by $C_{0}\left[u_{1}, C_{1}\left\{u_{2}, u_{3}\right\}, C_{2}\left\{u_{4}, C_{3}\left(u_{5}, u_{6}, u_{7}\right)\right\}\right]$, where $C_{k}$ is generated by the completely monotone generator $\psi_{k}, k \in\{0,1,2,3\}$. For this case, the sufficient nesting condition requires the appearing nodes $\psi_{0}^{-1} \circ \psi_{1}, \psi_{0}^{-1} \circ \psi_{2}$ and $\psi_{2}^{-1} \circ \psi_{3}$ in NAC to have completely monotone derivatives; see [15] for examples and general results when this holds. This implies that the functions $\psi_{k \ell}(t ; v)=\exp \left[-v \psi_{k}^{-1}\left\{\psi_{\ell}(t)\right\}\right], t \in[0, \infty), v \in(0, \infty)$, for $(k, \ell)=(0,1),(k, \ell)=(0,2)$ and $(k, \ell)=(2,3)$ are completely monotone generators for every $v$; see [12, p. 441]. As such, by Bernstein's Theorem, they correspond to distribution functions on the positive real line. The important part now is that if the frailties $V_{0}, V_{01}, V_{02}$ and $V_{23}$ are chosen level-by-level such that

1. $V_{0} \sim F_{0}=\mathcal{L S}^{-1}\left[\psi_{0}\right]$;

2. $V_{01} \mid V_{0} \sim F_{01}=\mathcal{L S}^{-1}\left[\psi_{01}\left(\cdot ; V_{0}\right)\right]$ and $V_{02} \mid V_{0} \sim F_{02}=\mathcal{L S}^{-1}\left[\psi_{02}\left(\cdot ; V_{0}\right)\right] ;$ and

3. $V_{23} \mid V_{02} \sim F_{23}=\mathcal{L S}^{-1}\left[\psi_{23}\left(\cdot ; V_{02}\right)\right]$,

then, by following along the lines as described in [17], one can show that the corresponding HAXC has the stochastic representation

$$
\boldsymbol{U}=\left(\psi_{0}\left(E_{1} / V_{0}\right), \psi_{1}\left(E_{2} V_{01}\right), \psi_{1}\left(E_{3} V_{01}\right), \psi_{2}\left(E_{4} V_{02}\right), \psi_{3}\left(E_{5} V_{23}\right), \psi_{3}\left(E_{6} V_{23}\right), \psi_{3}\left(E_{7} V_{23}\right)\right) .
$$

By comparison with (14), we see that if the distribution functions $F_{0}, F_{01}, F_{02}, F_{23}$ of $V_{0} \sim F_{0}, V_{01} \sim F_{01}\left(\cdot ; V_{0}\right)$, $V_{02} \sim F_{02}\left(\cdot ; V_{0}\right), V_{23} \sim F_{23}\left(\cdot ; V_{02}\right)$ are chosen such that the Laplace-Stieltjes transforms $\psi_{0}, \psi_{1}, \psi_{2}, \psi_{3}$ (associated to $V_{0}, V_{01}, V_{02}, V_{23}$ via the structure of a NAC) satisfy the sufficient nesting condition, then the marginal survival functions of (14) are not only known, but they are equal to $\psi_{0}, \psi_{1}, \psi_{2}, \psi_{3}$ such that the resulting HAXC has a stochastic representation (see (15)) similar to that of a HAXC with single frailty (see (13)), just with different frailties.

Remark 1. (i) Clearly, the stochastic representation of a HAXC based on hierarchical frailties as in (15) immediately allows for a sampling algorithm. The hierarchical frailties involved can easily be sampled in many cases, see [15] or the $\mathrm{R}$ package copula of [19] for details. 
(ii) Note that the stochastic representation of a HAXC constructed with hierarchical frailties equals that of a NAC, except for the fact that for the latter, the EVC $D$ of $\left(E_{1}, \ldots, E_{7}\right)$ is the independence copula.

(iii) The two types of constructing HAXCs presented here can also be mixed, one can use a HEVC and hierarchical frailties. Interestingly, the two types of hierarchies introduced this way do not have to coincide; see the following section for such an example.

The figures shown in the following examples can all be reproduced with the vignette HAXC of the R package copula (version $\geq 0.999 .19$ ).

Example 6 (ACs vs AXCs vs NACs vs (different) HAXCs). Figure 3 shows scatter-plot matrices of five-dimensional copula samples of size 1000 from the following models for $\boldsymbol{U}=\left(U_{1}, \ldots, U_{5}\right) \sim C$.

1. Top left: (Archimedean) Clayton copula with stochastic representation

$$
\boldsymbol{U}=\left(\psi\left(E_{1} / V\right), \ldots, \psi\left(E_{5} / V\right)\right),
$$

where $V \sim \Gamma(1 / \theta, 1)$ for $\theta=4 / 3$ (the frailty is Gamma distributed) and $E_{1}, \ldots, E_{5} \stackrel{\text { ind. }}{\sim} \mathcal{E}(1)$; see also (2). The copula parameter is chosen such that Kendall's tau equals 0.4 .

2. Top right: AXC based on Clayton's family with Gamma frailties recycled from the top left plot and stochastic representation as in $(16)$ where $\left(E_{1}, \ldots, E_{5}\right)=\left(-\ln Y_{1}, \ldots,-\ln Y_{5}\right)$ for $\left(Y_{1}, \ldots, Y_{5}\right)$ having a Gumbel EVC (with parameter such that Kendall's tau equals 0.5); note that the margins of $\left(E_{1}, \ldots, E_{5}\right)$ are again $\mathcal{E}(1)$ (but its components are dependent in this case).

3. Middle left: NAC based on Clayton's family with hierarchical frailties such that two sectors of sizes 2 and 3 result, respectively, with parameters $\left(\theta_{0}, \theta_{1}, \theta_{2}\right)$ chosen such that Kendall's tau equals 0.2 between the two sectors, 0.4 within the first sector and 0.6 within the second sector. A stochastic representation for this copula is given by

$$
\boldsymbol{U}=\left(\psi_{1}\left(E_{1} / V_{01}\right), \psi_{1}\left(E_{2} / V_{01}\right), \psi_{2}\left(E_{3} / V_{02}\right), \psi_{2}\left(E_{4} / V_{02}\right), \psi_{2}\left(E_{5} / V_{02}\right)\right),
$$

where $V_{0} \sim \Gamma(2)$ and

$$
\begin{aligned}
V_{01} \mid V_{0} \sim F_{01} & =\mathcal{L S}^{-1}\left[\exp \left[-V_{0}\left\{(1+t)^{\theta_{0} / \theta_{1}}-1\right\}\right]\right], \\
V_{02} \mid V_{0} \sim F_{02} & =\mathcal{L S}^{-1}\left[\exp \left[-V_{0}\left\{(1+t)^{\theta_{0} / \theta_{2}}-1\right\}\right]\right]
\end{aligned}
$$

are independent (see Theorem 3.6 in [16] for more details) and $E_{1}, \ldots, E_{5} \stackrel{\text { ind. }}{\sim} \mathcal{E}(1)$.

4. Middle right: HAXC based on Clayton's family with hierarchical frailties recycled from the middle left plot and stochastic representation as in $(17)$ where $\left(E_{1}, \ldots, E_{5}\right)=\left(-\ln Y_{1}, \ldots,-\ln Y_{5}\right)$ for $\left(Y_{1}, \ldots, Y_{5}\right)$ having a Gumbel EVC (realizations recycled from the top right plot). Note that the hierarchical structure is only induced by the frailties in this case.

5. Bottom left: HAXC based on Clayton's family with hierarchical frailties recycled from the middle left plot and stochastic representation

$$
\boldsymbol{U}=\left(\psi_{1}\left(E_{11} / V_{01}\right), \psi_{1}\left(E_{12} / V_{01}\right), \psi_{2}\left(E_{21} / V_{02}\right), \psi_{2}\left(E_{22} / V_{02}\right), \psi_{2}\left(E_{23} / V_{02}\right)\right),
$$

where

$$
\left(E_{11}, E_{12}, E_{21}, E_{22}, E_{23}\right)=\left(-\ln Y_{11},-\ln Y_{12},-\ln Y_{21},-\ln Y_{22},-\ln Y_{23}\right)
$$

for $\left(Y_{11}, Y_{12}, Y_{21}, Y_{22}, Y_{23}\right)$ having a nested Gumbel EVC (with sector sizes 2 and 3 and parameters such that Kendall's tau equals 0.2 between the two sectors, 0.5 within the first sector and 0.7 within the second sector). Note that the hierarchical structure is induced both at the level of the frailties and at the level of the EVC in this case, that the hierarchical structure (sectors, sector dimensions) is the same. 
6. Bottom right: $\mathrm{HAXC}$ as in the bottom left plot (realizations recycled) with stochastic representation

$$
\boldsymbol{U}=\left(\psi_{1}\left(E_{11} / V_{01}\right), \psi_{1}\left(E_{12} / V_{01}\right), \psi_{1}\left(E_{21} / V_{01}\right), \psi_{2}\left(E_{22} / V_{02}\right), \psi_{2}\left(E_{23} / V_{02}\right)\right) \text {. }
$$

Note that the hierarchical structure for the frailties (sector sizes 3 and 2, respectively) and for the nested Gumbel EVC (sector sizes 2 and 3, respectively) differ in this case.

Example 7 (EVCs vs HEVCs vs (different) HAXCs). Similar to Figure 3, Figure 4 shows scatter-plot matrices of five-dimensional copula samples of size 1000 from the following models for $\boldsymbol{U}=\left(U_{1}, \ldots, U_{5}\right) \sim C$; for simulating from the extremal $t \mathrm{EVC}$, we use the $\mathrm{R}$ package mev of [3].

1. Top left: Extremal $t \mathrm{EVC}$ with $\nu=3.5$ degrees of freedom and homogeneous correlation matrix $P$ with off-diagonal entries 0.7 .

2. Top right: Extremal $t$ HEVC with two sectors of sizes 2 and 3, respectively, such that the correlation matrix $P$ has entries 0.2 for pairs belonging to different sectors, 0.5 for pairs belonging to the first sector and 0.7 for pairs belonging to the second sector.

3. Middle left: HAXC with single Clayton frailty (as in Example 6 Part 1 ) and extremal $t$ HEVC recycled from the top right plot.

4. Middle right: HAXC with hierarchical Clayton frailties (as in Example 6 Part 3 ) and extremal $t$ EVC recycled from the top left plot.

5. Bottom left: HAXC with hierarchical Clayton frailties (as in Example 6 Part 3) and extremal $t$ HEVC recycled from the top right plot. Note that there are two types of hierarchies involved, at the level of the (hierarchical) frailties and at the level of the (hierarchical) extremal $t$ EVC. Furthermore, the two hierarchical structures match.

6. Bottom right: HAXC as in the bottom left plot, but the hierarchical structures of the frailties (sector sizes 3 and 2, respectively) and of the HEVC (sector sizes 2 and 3, respectively) differ in this case.

Note that we can sample from a hierarchical Schlather model (special case of extremal $t$ for $\nu=1$ ), a hierarchical Brown-Resnick model, their corresponding HAXCs in a similar fashion.

\section{Conclusion}

We extended the class of AXCs to HAXCs. Hierarchies can take place in two forms, either separately or simultaneously. First, the EVC involved in the construction of AXCs can have a hierarchical structure. To this end we presented a new approach for constructing hierarchical stable tail dependence functions based on a connection between stable tail dependence functions and $d$-norms. Second, a hierarchical structure can be imposed at the level of frailties similarly as NACs arise from ACs. Even more flexible constructions can be obtained by choosing a different hierarchical structure for the HEVC and the hierarchical frailties in the construction. Since all presented constructions are based on stochastic representations, sampling is immediate; see also the presented examples and vignette.

As a contribution to the literature on AXCs, we also derived a general formula for the density of AXCs and the computation of the corresponding logarithmic density. Furthermore, we briefly addressed the question when nested AXCs (NAXCs) can be constructed (either through nested stable tail dependence functions alone or, additionally, through hierarchical frailties). This is, in principle, possible, but there is currently only one family of examples known when all the assumptions involved are fulfilled. Further research is thus required to find out whether this is the only possible case for which NAXCs result. 

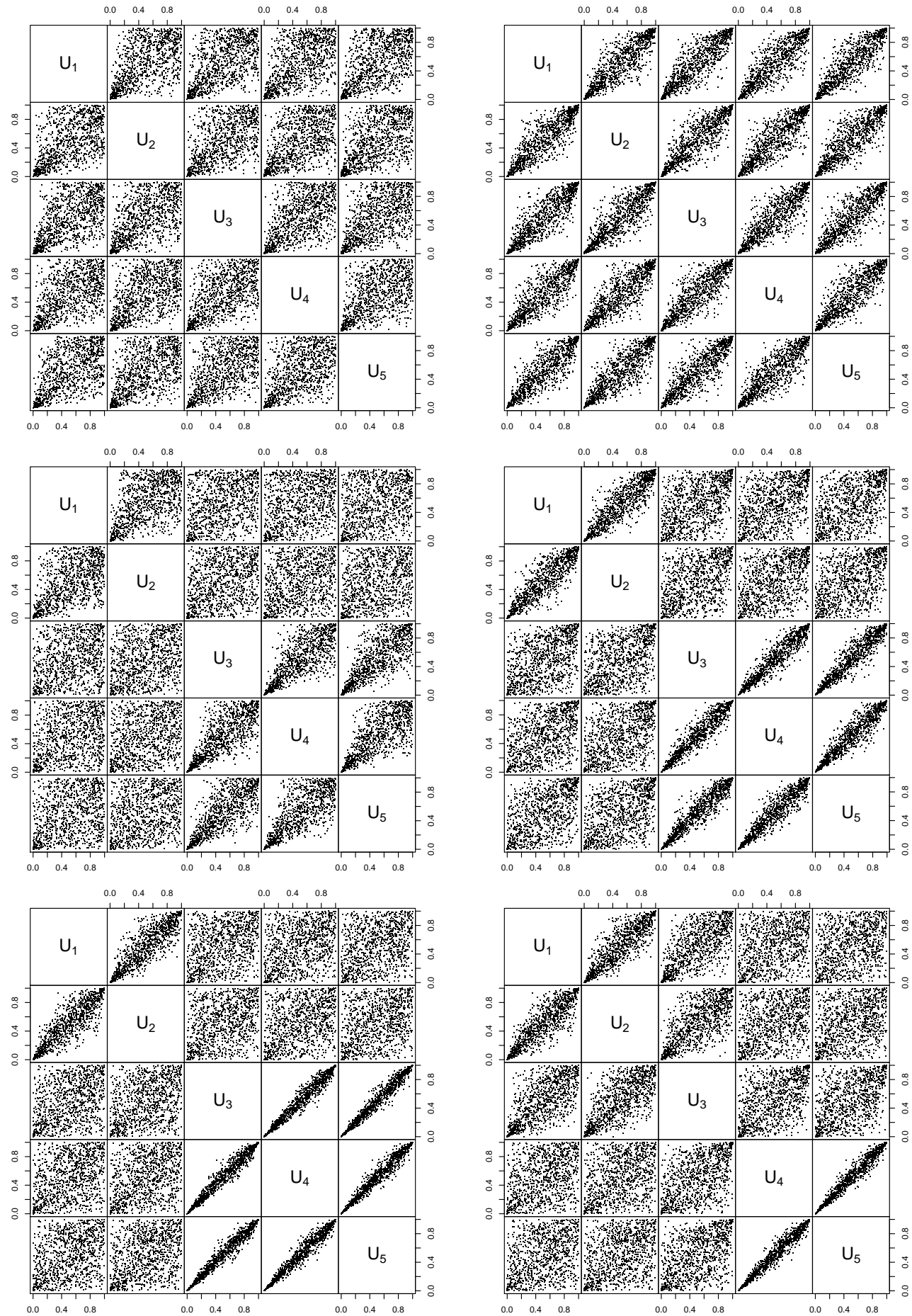

Figure 3: Scatter-plot matrices of five-dimensional copula samples of size 1000 of a Clayton copula (top left), an AXC with Clayton frailties and Gumbel EVC (top right), a nested Clayton copula (middle left), a HAXC with hierarchical Clayton frailties and Gumbel EVC (middle right), a HAXC with hierarchical Clayton frailties and nested Gumbel EVC of the same hierarchical structure (bottom left) and a HAXC with hierarchical Clayton frailties and nested Gumbel EVC of different hierarchical structure (bottom right). 

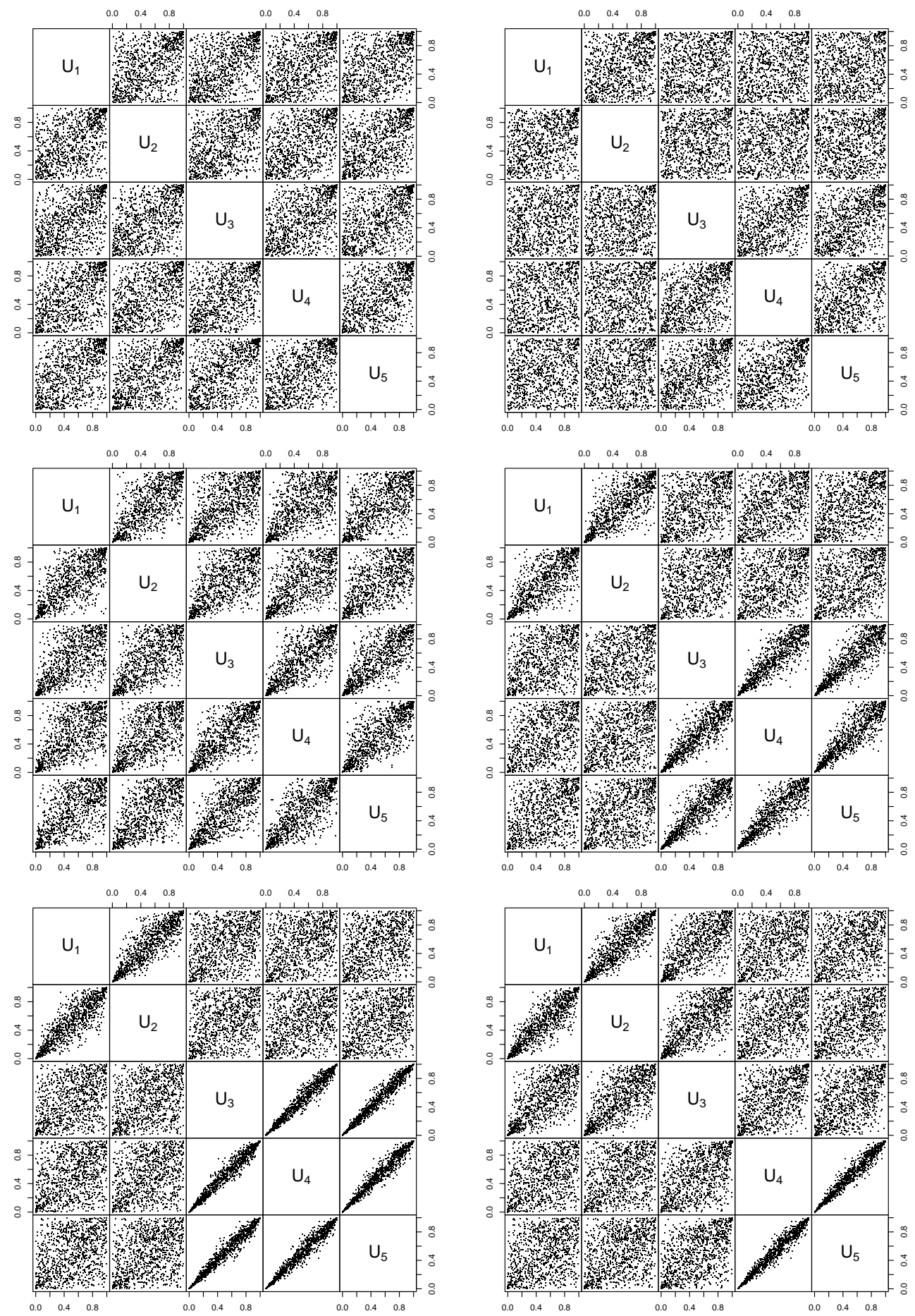

Figure 4: Scatter-plot matrices of five-dimensional copula samples of size 1000 of an extremal $t$ EVC (top left), a hierarchical extremal $t$ copula (a HEVC; top right), a HAXC with single Clayton frailty and extremal $t$ HEVC (middle left), a HAXC with hierarchical Clayton frailties and extremal $t$ EVC (middle right), a HAXC with hierarchical Clayton frailties and extremal $t$ HEVC of the same hierarchical structure (bottom left) and a HAXC with hierarchical Clayton frailties and extremal $t$ HEVC of different hierarchical structure (bottom right). 


\section{Acknowledgments}

The first author acknowledges support from NSERC (Grant RGPIN-5010-2015) and FIM, ETH Zürich. The third author acknowledges support from NSERC (PGS D scholarship). We would also like to thank the Editor-in-Chief, Christian Genest, the Associate Editor and the reviewers for their comments which helped to improve the paper substantially.

\section{Appendix A: On nested Archimax copulas}

We now briefly explore the question whether, in principle, HAXCs can also be nested copulas so nested Archimax copulas (NAXCs), i.e., whether there are HAXCs $C$ with analytical form $C(\boldsymbol{u})=$ $C_{0}\left\{C_{1}\left(\boldsymbol{u}_{1}\right), \ldots, C_{S}\left(\boldsymbol{u}_{S}\right)\right\}$ for all $\boldsymbol{u} \in[0,1]^{d}$. Note that the only known nontrivial class of copulas for which such nesting can be done (under the sufficient nesting condition) is the class of nested Archimedean copulas. To this end, we make the following assumption.

Assumption 1 (Nested EVCs). Assume that $D_{0}, \ldots, D_{S}$ are EVCs such that $D(\boldsymbol{u})=D_{0}\left\{D_{1}\left(\boldsymbol{u}_{1}\right), \ldots\right.$, $\left.D_{S}\left(\boldsymbol{u}_{S}\right)\right\}$ for all $\boldsymbol{u}=\left(\boldsymbol{u}_{1}, \ldots, \boldsymbol{u}_{S}\right) \in[0,1]^{d}$, is an $E V C$.

A function $D$ as in Assumption 1 is referred to as nested extreme-value copula (NEVC). The only known nontrivial copula family for which Assumption 1 is known to hold is the nested Gumbel family (under the sufficient nesting condition). It thus remains an open question whether there are other families of EVCs or a general construction of NEVCs besides the Gumbel.

\section{A.1 Based on nested extreme-value copulas or nested stable tail dependence functions}

Our first result shows that Assumption 1 is equivalent to the existence of a nested stable tail dependence function.

Lemma 1 (Nesting correspondence). An EVC D is a NEVC if and only if the stable tail dependence function $\ell$ of $D$ is nested, i.e.,

$$
\ell(\boldsymbol{x})=\ell_{0}\left\{\ell_{1}\left(\boldsymbol{x}_{1}\right), \ldots, \ell_{S}\left(\boldsymbol{x}_{S}\right)\right\}, \quad \boldsymbol{x} \in[0, \infty)^{d} .
$$

Proof. One has

$$
\begin{aligned}
D(\boldsymbol{u})= & D_{0}\left\{D_{1}\left(\boldsymbol{u}_{1}\right), \ldots, D_{S}\left(\boldsymbol{u}_{S}\right)\right\}=\exp \left[-\ell_{0}\left\{-\ln D_{1}\left(\boldsymbol{u}_{1}\right), \ldots,-\ln D_{S}\left(\boldsymbol{u}_{S}\right)\right\}\right] \\
= & \exp \left[-\ell_{0}\left[-\ln \left[\exp \left\{-\ell_{1}\left(-\ln u_{11}, \ldots,-\ln u_{1 d_{1}}\right)\right\}\right], \ldots,\right.\right. \\
& \left.\left.\quad-\ln \left[\exp \left\{-\ell_{S}\left(-\ln u_{S 1}, \ldots,-\ln u_{S d_{S}}\right)\right\}\right]\right]\right] \\
= & \exp \left[-\ell_{0}\left\{\ell_{1}\left(-\ln u_{11}, \ldots,-\ln u_{1 d_{1}}\right), \ldots, \ell_{S}\left(-\ln u_{S 1}, \ldots,-\ln u_{S d_{S}}\right)\right\}\right] \\
= & \exp \left\{-\ell\left(-\ln u_{11}, \ldots,-\ln u_{S d_{S}}\right)\right\}
\end{aligned}
$$

for all $\boldsymbol{u} \in[0,1]^{d}$ if and only if $\ell(\boldsymbol{x})=\ell_{0}\left\{\ell_{1}\left(\boldsymbol{x}_{1}\right), \ldots, \ell_{S}\left(\boldsymbol{x}_{S}\right)\right\}$ for all $\boldsymbol{x} \in[0, \infty)^{d}$.

The following proposition is essentially a nested version of one of the two HAXC extensions suggested in Section 3.2 which, based on Assumption 1 leads to nested AXCs (NAXCs) based on NEVCs or, equivalently, nested stable tail dependence functions; see Lemma 1.

Proposition 1 (NAXCs based on NEVCs or nested stable tail dependence functions). Let $D_{s}, s \in\{0, \ldots, S\}$, be as in Assumption 1 with respective stable tail dependence functions $\ell_{s}, s \in\{0, \ldots, S\}$. Let $V \sim F=$ $\mathcal{L S}^{-1}(\psi)$ and $\boldsymbol{Y}=\left(\boldsymbol{Y}_{1}, \ldots, \boldsymbol{Y}_{S}\right)=\left(Y_{11}, \ldots, Y_{1 d_{1}}, \ldots, Y_{S 1}, \ldots, Y_{S d_{S}}\right) \sim D$ be independent, where $D$ is an $E V C$ as in Assumption 1. Then the copula $C$ of

$$
\begin{aligned}
\boldsymbol{U} & =\left(\psi\left\{-\ln \left(\boldsymbol{Y}_{1}\right) / V\right\}, \ldots, \psi\left\{-\ln \left(\boldsymbol{Y}_{S}\right) / V\right\}\right) \\
& =\left(\psi\left\{-\ln \left(Y_{11}\right) / V\right\}, \ldots, \psi\left\{-\ln \left(Y_{1 d_{1}}\right) / V\right\}, \ldots, \psi\left\{-\ln \left(Y_{S 1}\right) / V\right\}, \ldots, \psi\left\{-\ln \left(Y_{S d_{S}}\right) / V\right\}\right)
\end{aligned}
$$


is given, for all $\boldsymbol{u} \in[0,1]^{d}$, by

$$
\begin{aligned}
C(\boldsymbol{u}) & =\psi\left[\ell_{0}\left[\ell_{1}\left\{\psi^{-1}\left(\boldsymbol{u}_{1}\right)\right\}, \ldots, \ell_{S}\left\{\psi^{-1}\left(\boldsymbol{u}_{S}\right)\right\}\right]\right] \\
& =\psi\left[\ell_{0}\left[\ell_{1}\left\{\psi^{-1}\left(u_{11}\right), \ldots, \psi^{-1}\left(u_{1 d_{1}}\right)\right\}, \ldots, \ell_{S}\left\{\psi^{-1}\left(u_{S 1}\right), \ldots, \psi^{-1}\left(u_{S d_{S}}\right)\right\}\right]\right] ;
\end{aligned}
$$

i.e., $C$ is an $A X C$ with nested stable tail dependence function as given in (A.1).

Proof. One has

$$
\begin{aligned}
\operatorname{Pr}(\boldsymbol{U} \leq \boldsymbol{u}) & =\operatorname{Pr}\left\{\boldsymbol{Y}_{1} \leq e^{-V \psi^{-1}\left(\boldsymbol{u}_{1}\right)}, \ldots, \boldsymbol{Y}_{S} \leq e^{-V \psi^{-1}\left(\boldsymbol{u}_{S}\right)}\right\} \\
& =\mathrm{E}\left[\operatorname{Pr}\left\{\boldsymbol{Y}_{1} \leq e^{-V \psi^{-1}\left(\boldsymbol{u}_{1}\right)}, \ldots, \boldsymbol{Y}_{S} \leq e^{-V \psi^{-1}\left(\boldsymbol{u}_{S}\right)} \mid V\right\}\right] \\
& =\mathrm{E}\left[D\left\{e^{-V \psi^{-1}\left(\boldsymbol{u}_{1}\right)}, \ldots, e^{-V \psi^{-1}\left(\boldsymbol{u}_{S}\right)}\right\}\right]=\mathrm{E}\left[D^{V}\left\{e^{-\psi^{-1}\left(\boldsymbol{u}_{1}\right)}, \ldots, e^{-\psi^{-1}\left(\boldsymbol{u}_{S}\right)}\right\}\right] \\
& =\mathrm{E}\left[\exp \left[-V \ell\left\{\psi^{-1}\left(\boldsymbol{u}_{1}\right), \ldots, \psi^{-1}\left(\boldsymbol{u}_{S}\right)\right\}\right]\right]=\psi\left[\ell\left\{\psi^{-1}\left(\boldsymbol{u}_{1}\right), \ldots, \psi^{-1}\left(\boldsymbol{u}_{S}\right)\right\}\right]
\end{aligned}
$$

The claim immediately follows from Lemma 1 by noting that $D$ is nested as of Assumption 1 .

Corollary 1 (Pairwise marginal copulas). Under the setup of Proposition 1 the bivariate marginal copulas of $C$ satisfy

$$
C\left(u_{s i}, u_{t j}\right)= \begin{cases}\psi\left[\ell_{s}\left\{\psi^{-1}\left(u_{s i}\right), \psi^{-1}\left(u_{s j}\right)\right\}\right] & \text { if } t=s, \\ \psi\left[\ell_{0}\left\{\psi^{-1}\left(u_{s i}\right), \psi^{-1}\left(u_{t j}\right)\right\}\right] & \text { otherwise }\end{cases}
$$

Therefore, the bivariate marginal copulas of $C$ are (possibly different) AXCs.

Proof. For a stable tail dependence function $\ell$, one has that $\ell(\boldsymbol{x})=x_{j}$ if all components except the $j$ th of $\boldsymbol{x}$ are 0 . As such, for any $s \in\{1, \ldots, S\}$,

$$
\ell_{s}\left\{\psi^{-1}\left(u_{s 1}\right), \ldots, \psi^{-1}\left(u_{s d_{s}}\right)\right\}= \begin{cases}0, & \text { if } u_{s j}=1 \text { for all } j \in\left\{1, \ldots, d_{s}\right\}, \\ \psi^{-1}\left(u_{s k}\right), & \text { if } u_{s j}=1 \text { for all } j \in\left\{1, \ldots, d_{s}\right\} \backslash\{k\}, \\ \ell_{s}\left\{\psi^{-1}\left(u_{s k}\right), \psi^{-1}\left(u_{s \ell}\right)\right\}, & \text { if } u_{s j}=1 \text { for all } j \in\left\{1, \ldots, d_{s}\right\} \backslash\{k, \ell\},\end{cases}
$$

from which the result follows.

\section{A.2 Additionally nesting frailties}

As in the second method for introducing hierarchies on AXCs presented in Section 3.2, we could, additionally, impose a hierarchical structure on the underlying (multiple) frailties. We focus on the two-level case with $S$ different frailties. Assume, as before, the sufficient nesting condition to hold, i.e., $\psi_{s} \in \Psi$, $s \in\{0, \ldots, S\}$, are Archimedean generators and, for all $s \in\{0, \ldots, S\}$, the derivative of $\psi_{0}^{-1} \circ \psi_{s}$ is completely monotone.

Proposition 2 (NAXCs based on nested frailties). Let $D_{s}, s \in\{0, \ldots, S\}$, be as in Assumption 1 with respective stable tail dependence functions $\ell_{s}, s \in\{0, \ldots, S\}$. Furthermore, let $\psi_{s} \in \Psi$ be completely monotone, $s \in\{0, \ldots, S\}$, assume that the sufficient nesting condition holds. Assume $V_{0} \sim F_{0}=\mathcal{L S}^{-1}\left[\psi_{0}\right]$ and $V_{0 s} \mid V_{0} \sim F_{0 s}=\mathcal{L S}^{-1}\left\{\psi_{0 s}\left(\cdot ; V_{0}\right)\right\}, s \in\{1, \ldots, S\}$. Moreover, let $\boldsymbol{Y}=\left(\boldsymbol{Y}_{1}, \ldots, \boldsymbol{Y}_{S}\right) \sim D$ be independent of $V_{0}, \ldots, V_{S}$ and assume that

$$
\begin{aligned}
\mathrm{E}\left\{\mathrm { E } \left(D _ { 0 } \left[D_{1}\left\{e^{-V_{01} \psi_{1}^{-1}\left(\boldsymbol{u}_{1}\right)}\right\}, \ldots\right.\right.\right. & \left.\left.\left., D_{S}\left\{e^{-V_{0 S} \psi_{S}^{-1}\left(\boldsymbol{u}_{S}\right)}\right\}\right] \mid V_{0}\right)\right\} \\
& =\mathrm{E}\left\{D_{0}\left(\mathrm{E}\left[D_{1}\left\{e^{-V_{01} \psi_{1}^{-1}\left(\boldsymbol{u}_{1}\right)}\right\} \mid V_{0}\right], \ldots, \mathrm{E}\left[D_{S}\left\{e^{-V_{0 S} \psi_{S}^{-1}\left(\boldsymbol{u}_{S}\right)}\right\} \mid V_{0}\right]\right)\right\} .
\end{aligned}
$$

Then the copula $C$ of $\boldsymbol{U}=\left(\psi_{1}\left\{-\ln \left(\boldsymbol{Y}_{1}\right) / V_{01}\right\}, \ldots, \psi_{S}\left\{-\ln \left(\boldsymbol{Y}_{S}\right) / V_{0 S}\right\}\right)$ is given, for all $\boldsymbol{u} \in[0,1]^{d}$, by $C(\boldsymbol{u})=C_{0}\left\{C_{1}\left(\boldsymbol{u}_{1}\right), \ldots, C_{S}\left(\boldsymbol{u}_{S}\right)\right\}$, where, for all $s \in\{0, \ldots, S\}, C_{s}$ is Archimax with generator $\psi_{s}$ and stable tail dependence function $\ell_{s}$. 
Proof. One has

$$
\begin{aligned}
\operatorname{Pr}(\boldsymbol{U} \leq \boldsymbol{u}) & =\operatorname{Pr}\left\{\boldsymbol{Y}_{1} \leq e^{-V_{01} \psi_{1}^{-1}\left(\boldsymbol{u}_{1}\right)}, \ldots, \boldsymbol{Y}_{S} \leq e^{-V_{0 S} \psi_{S}^{-1}\left(\boldsymbol{u}_{S}\right)}\right\} \\
& =\mathrm{E}\left(\mathrm{E}\left[\operatorname{Pr}\left\{\boldsymbol{Y}_{1} \leq e^{-V_{01} \psi_{1}^{-1}\left(\boldsymbol{u}_{1}\right)}, \ldots, \boldsymbol{Y}_{S} \leq e^{-V_{0 S} \psi_{S}^{-1}\left(\boldsymbol{u}_{S}\right)} \mid V_{01}, \ldots, V_{0 S}\right\} \mid V_{0}\right]\right) \\
& =\mathrm{E}\left(\mathrm{E}\left[D\left\{e^{-V_{01} \psi_{1}^{-1}\left(\boldsymbol{u}_{1}\right)}, \ldots, e^{-V_{0 S} \psi_{S}^{-1}\left(\boldsymbol{u}_{S}\right)}\right\} \mid V_{0}\right]\right) \\
& =\mathrm{E}\left\{D_{0}\left(\mathrm{E}\left[D_{1}\left\{e^{-V_{01} \psi_{1}^{-1}\left(\boldsymbol{u}_{1}\right)}\right\} \mid V_{0}\right], \ldots, \mathrm{E}\left[D_{S}\left\{e^{-V_{0 S} \psi_{S}^{-1}\left(\boldsymbol{u}_{S}\right)}\right\} \mid V_{0}\right]\right)\right\} .
\end{aligned}
$$

Each component $\mathrm{E}\left[D_{s}\left\{e^{-V_{0 s} \psi_{s}^{-1}\left(\boldsymbol{u}_{s}\right)}\right\} \mid V_{0}\right], s \in\{1, \ldots, S\}$, satisfies

$$
\begin{aligned}
\mathrm{E}\left[D_{s}\left\{e^{-V_{0 s} \psi_{s}^{-1}\left(\boldsymbol{u}_{s}\right)}\right\} \mid V_{0}\right] & =\mathrm{E}\left[D_{s}^{V_{0 s}}\left\{e^{-\psi_{s}^{-1}\left(\boldsymbol{u}_{s}\right)}\right\} \mid V_{0}\right]=\mathrm{E}\left[e^{-V_{0 s} \ell_{s}\left\{\psi_{s}^{-1}\left(\boldsymbol{u}_{s}\right)\right\}} \mid V_{0}\right] \\
& =\psi_{0 s}\left[\ell_{s}\left\{\psi_{s}^{-1}\left(\boldsymbol{u}_{s}\right)\right\} ; V_{0}\right],
\end{aligned}
$$

thus

$$
\begin{aligned}
\operatorname{Pr}(\boldsymbol{U} \leq \boldsymbol{u}) & =\mathrm{E}\left\{D_{0}\left(\psi_{01}\left[\ell_{1}\left\{\psi_{1}^{-1}\left(\boldsymbol{u}_{1}\right)\right\} ; V_{0}\right], \ldots, \psi_{0 S}\left[\ell_{S}\left\{\psi_{S}^{-1}\left(\boldsymbol{u}_{S}\right)\right\} ; V_{0}\right]\right)\right\} \\
& =\mathrm{E}\left(D_{0}\left[e^{-V_{0} \psi_{0}^{-1}\left\{C_{1}\left(\boldsymbol{u}_{1}\right)\right\}}, \ldots, e^{-V_{0} \psi_{0}^{-1}\left\{C_{S}\left(\boldsymbol{u}_{S}\right)\right\}}\right]\right) \\
& =\mathrm{E}\left(D_{0}^{V_{0}}\left[e^{-\psi_{0}^{-1}\left\{C_{1}\left(\boldsymbol{u}_{1}\right)\right\}}, \ldots, e^{-\psi_{0}^{-1}\left\{C_{S}\left(\boldsymbol{u}_{S}\right)\right\}}\right]\right) \\
& =\mathrm{E}\left\{e^{-V_{0}\left(\ell_{0}\left[\psi_{0}^{-1}\left\{C_{1}\left(\boldsymbol{u}_{1}\right)\right\}, \ldots, \psi_{0}^{-1}\left\{C_{S}\left(\boldsymbol{u}_{S}\right)\right\}\right]\right)}\right\} \\
& =\psi_{0}\left(\ell_{0}\left[\psi_{0}^{-1}\left\{C_{1}\left(\boldsymbol{u}_{1}\right)\right\}, \ldots, \psi_{0}^{-1}\left\{C_{S}\left(\boldsymbol{u}_{S}\right)\right\}\right]\right)=C_{0}\left\{C_{1}\left(\boldsymbol{u}_{1}\right), \ldots, C_{S}\left(\boldsymbol{u}_{S}\right)\right\} .
\end{aligned}
$$

The following corollary provides a condition under which Assumption (A.2) holds. Note that this particular model can already be found in [29].

Corollary 2 (AC composed with AXCs). If $D(\boldsymbol{u})=D_{1}\left(\boldsymbol{u}_{1}\right) \times \cdots \times D_{S}\left(\boldsymbol{u}_{S}\right)$ for all $\boldsymbol{u} \in[0,1]^{d}$, then $(\mathrm{A} .2)$ holds and $C(\boldsymbol{u})=C_{0}\left\{C_{1}\left(\boldsymbol{u}_{1}\right), \ldots, C_{S}\left(\boldsymbol{u}_{S}\right)\right\}$ for all $\boldsymbol{u} \in[0,1]^{d}$, where $C_{0}$ is Archimedean and $C_{1}, \ldots, C_{S}$ are Archimax. In particular, if $D$ is the independence copula, (A.2) holds and $C$ is a NAC.

Proof. If $D(\boldsymbol{u})=D_{1}\left(\boldsymbol{u}_{1}\right) \times \cdots \times D_{S}\left(\boldsymbol{u}_{S}\right)$, then, conditional on $V_{0}$, the sector components are independent and we obtain

$$
\begin{aligned}
& \mathrm{E}\left(D_{0}\left[D_{1}\left\{e^{-V_{01} \psi_{1}^{-1}\left(\boldsymbol{u}_{1}\right)}\right\}, \ldots, D_{S}\left\{e^{-V_{0 S} \psi_{S}^{-1}\left(\boldsymbol{u}_{S}\right)}\right\}\right] \mid V_{0}\right) \\
&=\mathrm{E}\left[\prod_{s=1}^{S} D_{s}\left\{e^{-V_{0 s} \psi_{s}^{-1}\left(\boldsymbol{u}_{s}\right)}\right\} \mid V_{0}\right]=\prod_{s=1}^{S} \mathrm{E}\left[D_{s}\left\{e^{-V_{0 s} \psi_{s}^{-1}\left(\boldsymbol{u}_{s}\right)}\right\} \mid V_{0}\right] \\
& \quad=D_{0}\left(\mathrm{E}\left[D_{1}\left\{e^{-V_{01} \psi_{1}^{-1}\left(\boldsymbol{u}_{1}\right)}\right\} \mid V_{0}\right], \ldots, \mathrm{E}\left[D_{S}\left\{e^{-V_{0 S} \psi_{S}^{-1}\left(\boldsymbol{u}_{S}\right)}\right\} \mid V_{0}\right]\right) .
\end{aligned}
$$

and thus (A.2) follows by taking the expectation. The rest follows immediately by noting that an EVC is the independence copula if and only if its stable tail dependence function is the sum of its components, so the Archimax (sector) copulas $C_{s}\left(\boldsymbol{u}_{s}\right)=\psi_{s}\left[\ell_{s}\left\{\psi_{s}^{-1}\left(u_{s 1}\right), \ldots, \psi_{s}^{-1}\left(u_{s d_{s}}\right)\right\}\right]$ are Archimedean generated by $\psi_{s}$, $s \in\{1, \ldots, S\}$.

\section{Appendix B. Density of Archimax copulas}

For likelihood-based inference on AXCs, it is important to know their density. In this section, we present the general form of the density of AXCs (if it exists) and address how it can be computed numerically.

Proposition 3 (AXC density). If the respective partial derivatives of $\ell$ exist and are continuous, the density $c$ of a d-dimensional $A X C C$ is given, for all $\boldsymbol{u} \in(0,1)^{d}$, by

$$
c(\boldsymbol{u})=\left\{\prod_{j=1}^{d}\left(\psi^{-1}\right)^{\prime}\left(u_{j}\right)\right\} \sum_{k=1}^{d} \psi^{(k)}\left[\ell\left\{\psi^{-1}(\boldsymbol{u})\right\}\right] \sum_{\pi \in \Pi:|\pi|=k} \prod_{B \in \pi}\left(\mathrm{D}_{B} \ell\right)\left\{\psi^{-1}(\boldsymbol{u})\right\},
$$


where $\psi^{-1}(\boldsymbol{u})=\left(\psi^{-1}\left(u_{1}\right), \ldots, \psi^{-1}\left(u_{d}\right)\right)$, $\Pi$ denotes the set of all partitions $\pi$ of $\{1, \ldots, d\}$ (with $|\pi|$ denoting the number of elements of $\pi)$ and $\left(\mathrm{D}_{B} \ell\right)\left\{\psi^{-1}(\boldsymbol{u})\right\}$ denotes the partial derivatives of $\ell$ with respect to the variables with index in $B$, evaluated at $\psi^{-1}(\boldsymbol{u})$.

Proof. By a multivariate version of Faà di Bruno's Formula, see [14], the $d$ th derivative of a composition of two functions $f: \mathbb{R} \rightarrow \mathbb{R}$ and $g: \mathbb{R}^{d} \rightarrow \mathbb{R}$ is given by

$$
\begin{aligned}
\mathrm{D} f\{g(\boldsymbol{x})\} & =\sum_{\pi \in \Pi}\left[f^{(|\pi|)}\{g(\boldsymbol{x})\} \prod_{B \in \pi} \mathrm{D}_{B} g(\boldsymbol{x})\right]=\sum_{k=1}^{d} \sum_{\pi \in \Pi:|\pi|=k}\left[f^{(|\pi|)}\{g(\boldsymbol{x})\} \prod_{B \in \pi} \mathrm{D}_{B} g(\boldsymbol{x})\right] \\
& =\sum_{k=1}^{d} \sum_{\pi \in \Pi:|\pi|=k}\left[f^{(k)}\{g(\boldsymbol{x})\} \prod_{B \in \pi} \mathrm{D}_{B} g(\boldsymbol{x})\right]=\sum_{k=1}^{d} f^{(k)}\{g(\boldsymbol{x})\} \sum_{\pi \in \Pi:|\pi|=k} \prod_{B \in \pi} \mathrm{D}_{B} g(\boldsymbol{x}),
\end{aligned}
$$

where $\mathrm{D}=\partial^{d} /\left(\partial x_{d} \cdots \partial x_{1}\right), \mathrm{D}_{B}=\partial^{|B|} / \prod_{j \in B} \partial x_{j}, B \in \pi$ means that $B$ runs through all partition elements of $\pi$. Assuming that the appearing derivatives exist and are continuous, we obtain from taking $f(x)=\psi(x)$ and $g(\boldsymbol{x})=\ell\left\{\psi^{-1}(\boldsymbol{x})\right\}$ that, for all $\boldsymbol{u} \in(0,1)^{d}$,

$$
\begin{aligned}
c(\boldsymbol{u}) & =\sum_{k=1}^{d} \psi^{(k)}\left[\ell\left\{\psi^{-1}(\boldsymbol{u})\right\}\right] \sum_{\pi \in \Pi:|\pi|=k} \prod_{B \in \pi} \frac{\partial^{|B|}}{\prod_{j \in B} \partial u_{j}} \ell\left\{\psi^{-1}(\boldsymbol{u})\right\} \\
& =\left\{\prod_{j=1}^{d}\left(\psi^{-1}\right)^{\prime}\left(u_{j}\right)\right\} \sum_{k=1}^{d} \psi^{(k)}\left[\ell\left\{\psi^{-1}(\boldsymbol{u})\right\}\right] \sum_{\pi \in \Pi:|\pi|=k} \prod_{B \in \pi}\left(\mathrm{D}_{B} \ell\right)\left\{\psi^{-1}(\boldsymbol{u})\right\},
\end{aligned}
$$

where the last equality holds since the derivatives of all of $\psi^{-1}\left(u_{1}\right), \ldots, \psi^{-1}\left(u_{d}\right)$ (from applying the chain rule) appear in each summand of the sum $\sum_{\pi \in \Pi:|\pi|=k}$ and can thus be taken out of both summations.

As a quick check of Proposition 3, we can recover the density of ACs and EVCs.

Corollary 3 (AC density as special case). For $\ell(\boldsymbol{x})=x_{1}+\cdots+x_{d}$, the density of ACs correctly follows from Proposition 3 by noting that

$$
\sum_{\pi \in \Pi:|\pi|=k} \prod_{B \in \pi}\left(\mathrm{D}_{B} \ell\right)(\boldsymbol{x})=\sum_{\pi \in \Pi:|\pi|=k} \prod_{B \in \pi} \mathbf{1}_{\{|B|=1\}}=\sum_{\pi \in \Pi:|\pi|=k} \mathbf{1}_{\{|B|=1 \text { for all } B \in \pi\}}=\mathbf{1}_{\{k=d\}} .
$$

Corollary 4 (EVC density as special case). For $\psi(t)=e^{-t}, t \geq 0$, the density of EVCs correctly follows from Proposition 3 as one has, for all $\boldsymbol{u} \in(0,1)^{d}$,

$$
c(\boldsymbol{u})=\left\{\prod_{j=1}^{d}\left(-\frac{1}{u_{j}}\right)\right\} \sum_{k=1}^{d} \exp [-\ell\{-\ln (\boldsymbol{u})\}] \sum_{\pi \in \Pi:|\pi|=k} \prod_{B \in \pi}\left[-\left(\mathrm{D}_{B} \ell\right)\{-\ln (\boldsymbol{u})\}\right] ;
$$

see, e.g., [6] or [11].

The following result provides the general form of the density of AXCs based on the stable tail dependence function $\ell$ of a Gumbel copula.

Corollary 5 (Density of AXCs with Gumbel stable tail dependence function as special case). For the stable tail dependence function of a Gumbel copula with parameter $\alpha \in(0,1]$, defined, for all $\boldsymbol{x} \in[0, \infty)^{d}$, by $\ell(\boldsymbol{x})=\left(x_{1}^{1 / \alpha}+\cdots+x_{d}^{1 / \alpha}\right)^{\alpha}$, the density $c$ of an $A X C$ is given by

$$
\begin{aligned}
c(\boldsymbol{u})= & \frac{1}{\alpha^{d}}\left\{\prod_{j=1}^{d}\left(\psi^{-1}\right)^{\prime}\left(u_{j}\right) \psi^{-1}\left(u_{j}\right)^{1 / \alpha-1}\right\} \\
& \times \sum_{k=1}^{d} \psi^{(k)}\left[\left\{\sum_{j=1}^{d} \psi^{-1}\left(u_{j}\right)^{1 / \alpha}\right\}^{\alpha}\right]\left\{\sum_{j=1}^{d} \psi^{-1}\left(u_{j}\right)^{1 / \alpha}\right\}^{\alpha k-d} \sum_{\pi \in \Pi:|\pi|=k} \prod_{B \in \pi}(\alpha)_{|B|}, \quad \boldsymbol{u} \in(0,1)^{d},
\end{aligned}
$$

where $(\alpha)_{|B|}=\prod_{\ell=0}^{|B|-1}(\alpha-\ell)$ denotes the falling factorial. 
Proof. For this specific stable tail dependence function, one has

$$
\mathrm{D}_{B} \ell(\boldsymbol{x})=(\alpha)_{|B|}\left(\sum_{j=1}^{d} x_{j}^{1 / \alpha}\right)^{\alpha-|B|}\left(\frac{1}{\alpha}\right)^{|B|} \prod_{j \in B} x_{j}^{1 / \alpha-1} .
$$

Since every index in $\{1, \ldots, d\}$ appears in precisely one $B \in \pi$,

$$
\begin{aligned}
\sum_{\pi \in \Pi:|\pi|=k} \prod_{B \in \pi} \mathrm{D}_{B} \ell(\boldsymbol{x}) & =\frac{1}{\alpha^{d}} \prod_{j=1}^{d} x_{j}^{1 / \alpha-1} \sum_{\pi \in \Pi:|\pi|=k} \prod_{B \in \pi}(\alpha)_{|B|}\left(\sum_{j=1}^{d} x_{j}^{1 / \alpha}\right)^{\alpha-|B|} \\
& =\frac{1}{\alpha^{d}} \prod_{j=1}^{d} x_{j}^{1 / \alpha-1}\left(\sum_{j=1}^{d} x_{j}^{1 / \alpha}\right)^{\alpha k-d} \sum_{\pi \in \Pi:|\pi|=k} \prod_{B \in \pi}(\alpha)_{|B|} .
\end{aligned}
$$

Using the general form of the density as given in Proposition 3 and $\boldsymbol{x}=\psi^{-1}(\boldsymbol{u})$ leads to the result as stated.

As we can see from Proposition 3, the general form of the density of AXCs involves the (possibly high-order) derivatives $\psi^{(k)}$ and $\mathrm{D}_{B} \ell$. The former are well known to be numerically non-trivial; see, e.g., $[20,21]$. We therefore now address how the density of AXCs can be computed numerically. This is typically done by computing a proper $\operatorname{logarithm}$, i.e., a logarithm which is numerically more robust than just $\ln c$, then returning the exponential (but only if required). As we will see, two nested proper logarithms can be used to evaluate the logarithmic density of AXCs, which is especially appealing.

Proposition 4 (AXC logarithmic density evaluation). If the respective partial derivatives of $\ell$ exist and are continuous, the logarithmic density $\ln c$ of a d-dimensional $A X C C$ is given, for all $\boldsymbol{u} \in(0,1)^{d}$, by

$$
\ln c(\boldsymbol{u})=\sum_{j=1}^{d} \ln \left\{\left(-\psi^{-1}\right)^{\prime}\left(u_{j}\right)\right\}+b_{m a x}^{\psi, \ell}(\boldsymbol{u})+\ln \sum_{k=1}^{d} \exp \left\{b_{k}^{\psi, \ell}(\boldsymbol{u})-b_{m a x}^{\psi, \ell}(\boldsymbol{u})\right\},
$$

where the notation is as in Proposition 3 and

$$
\begin{aligned}
& b_{k}^{\psi, \ell}(\boldsymbol{u})=\ln \left\{(-1)^{k} \psi^{(k)}\right\}\left[\ell\left\{\psi^{-1}(\boldsymbol{u})\right\}\right]+a_{\max }^{\psi, \ell, k}(\boldsymbol{u})+\ln \sum_{\pi \in \Pi:|\pi|=k} \exp \left\{a_{\pi}^{\psi, \ell, k}(\boldsymbol{u})-a_{m a x}^{\psi, \ell, k}(\boldsymbol{u})\right\}, \\
& b_{\max }^{\psi, \ell}(\boldsymbol{u})=\max _{k} b_{k}^{\psi, \ell}(\boldsymbol{u})
\end{aligned}
$$

for

$$
a_{\pi}^{\psi, \ell, k}(\boldsymbol{u})=\sum_{B \in \pi} \ln \left\{(-1)^{|B|-1} \mathrm{D}_{B} \ell\right\}\left\{\psi^{-1}(\boldsymbol{u})\right\}, \quad a_{\max }^{\psi, \ell, k}(\boldsymbol{u})=\max _{\pi \in \Pi:|\pi|=k} a_{\pi}^{\psi, \ell, k}(\boldsymbol{u}) .
$$

Proof. Let $\boldsymbol{u} \in(0,1)^{d}$ and note that

$$
\begin{aligned}
c(\boldsymbol{u}) & =\left\{\prod_{j=1}^{d}\left(\psi^{-1}\right)^{\prime}\left(u_{j}\right)\right\} \sum_{k=1}^{d} \psi^{(k)}\left[\ell\left\{\psi^{-1}(\boldsymbol{u})\right\}\right] \sum_{\pi \in \Pi:|\pi|=k} \prod_{B \in \pi}\left(\mathrm{D}_{B} \ell\right)\left\{\psi^{-1}(\boldsymbol{u})\right\} \\
& =\left\{\prod_{j=1}^{d}\left(-\psi^{-1}\right)^{\prime}\left(u_{j}\right)\right\} \sum_{k=1}^{d}(-1)^{k} \psi^{(k)}\left[\ell\left\{\psi^{-1}(\boldsymbol{u})\right\}\right] \sum_{\pi \in \Pi:|\pi|=k}(-1)^{d-k} \prod_{B \in \pi}\left(\mathrm{D}_{B} \ell\right)\left\{\psi^{-1}(\boldsymbol{u})\right\} \\
& =\left\{\prod_{j=1}^{d}\left(-\psi^{-1}\right)^{\prime}\left(u_{j}\right)\right\} \sum_{k=1}^{d}(-1)^{k} \psi^{(k)}\left[\ell\left\{\psi^{-1}(\boldsymbol{u})\right\}\right] \sum_{\pi \in \Pi:|\pi|=k} \prod_{B \in \pi}\left\{(-1)^{|B|-1} \mathrm{D}_{B} \ell\right\}\left\{\psi^{-1}(\boldsymbol{u})\right\},
\end{aligned}
$$


where the last equality follows from the fact that $\sum_{B \in \pi}|B|=d$ and $\prod_{B \in \pi} D_{B} \ell$ is taken over those $\pi$ for which $|\pi|=k$, so $\sum_{B \in \pi} 1=k$; note that, as before, $|B|$ denotes the number of elements of $B$.

Since $\psi$ has derivatives with alternating signs, $(-1)^{k} \psi^{(k)}>0$ for all arguments; in particular, $\left(-\psi^{-1}\right)^{\prime}>0$, too. By Theorem 6 in [39], $\ell$ is fully $d$-max decreasing which implies that, for all arguments of $\ell$, $\operatorname{sign}\left(\mathrm{D}_{B} \ell\right)=$ $(-1)^{|B|-1}$. This implies that $\operatorname{sign}\left\{(-1)^{|B|-1} \mathrm{D}_{B} \ell\right\}=1$ and so all terms $a_{\pi}^{\psi, \ell, k}$ and $b_{k}^{\psi, \ell}$ as defined in the claim are well-defined.

Taking the logarithm, the first product in $c$ becomes $\sum_{j=1}^{d} \ln \left\{\left(-\psi^{-1}\right)^{\prime}\left(u_{j}\right)\right\}$ as in the claim. By using the definitions in the claim, the logarithm of the remaining sum can be written as

$$
\ln \sum_{k=1}^{d} \exp \left[\ln \left\{(-1)^{k} \psi^{(k)}\left[\ell\left\{\psi^{-1}(\boldsymbol{u})\right\}\right] \sum_{\pi \in \Pi:|\pi|=k} \prod_{B \in \pi}\left\{(-1)^{|B|-1} \mathrm{D}_{B} \ell\right\}\left\{\psi^{-1}(\boldsymbol{u})\right\}\right\}\right],
$$

where

$$
\begin{aligned}
\ln \left[(-1)^{k} \psi^{(k)}\left[\ell\left\{\psi^{-1}(\boldsymbol{u})\right\}\right]\right. & \left.\sum_{\pi \in \Pi:|\pi|=k} \prod_{B \in \pi}\left\{(-1)^{|B|-1} \mathrm{D}_{B} \ell\right\}\left\{\psi^{-1}(\boldsymbol{u})\right\}\right] \\
& =\ln \left((-1)^{k} \psi^{(k)}\left[\ell\left\{\psi^{-1}(\boldsymbol{u})\right\}\right]\right)+\ln \sum_{\pi \in \Pi:|\pi|=k} \prod_{B \in \pi}\left\{(-1)^{|B|-1} \mathrm{D}_{B} \ell\right\}\left\{\psi^{-1}(\boldsymbol{u})\right\} \\
& =\ln \left\{(-1)^{k} \psi^{(k)}\right\}\left[\ell\left\{\psi^{-1}(\boldsymbol{u})\right\}\right]+\ln \sum_{\pi \in \Pi:|\pi|=k} \exp \left[\sum_{B \in \pi} \ln \left\{(-1)^{|B|-1} \mathrm{D}_{B} \ell\right\}\left\{\psi^{-1}(\boldsymbol{u})\right\}\right] \\
& =\ln \left\{(-1)^{k} \psi^{(k)}\right\}\left[\ell\left\{\psi^{-1}(\boldsymbol{u})\right\}\right]+\ln \sum_{\pi \in \Pi:|\pi|=k} \exp \left\{a_{\pi}^{\psi, \ell, k}(\boldsymbol{u})\right\} \\
& =\ln \left\{(-1)^{k} \psi^{(k)}\right\}\left[\ell\left\{\psi^{-1}(\boldsymbol{u})\right\}\right]+a_{\max }^{\psi, \ell, k}(\boldsymbol{u})+\ln \sum_{\pi \in \Pi:|\pi|=k} \exp \left\{a_{\pi}^{\psi, \ell, k}(\boldsymbol{u})-a_{\max }^{\psi, \ell, k}(\boldsymbol{u})\right\} \\
& =b_{k}^{\psi, \ell}(\boldsymbol{u}) .
\end{aligned}
$$

We thus obtain that the term in (B.1) equals

$$
\ln \sum_{k=1}^{d} \exp \left\{b_{k}^{\psi, \ell}(\boldsymbol{u})\right\}=b_{\max }^{\psi, \ell}(\boldsymbol{u})+\ln \sum_{k=1}^{d} \exp \left\{b_{k}^{\psi, \ell}(\boldsymbol{u})-b_{\max }^{\psi, \ell}(\boldsymbol{u})\right\} .
$$

Putting the terms together, the logarithmic density has the form as in the claim.

A couple of remarks are in order here. First, note that due to the signs of the involved terms, one can apply an (exp-ln)-trick twice (nested) for computing the logarithmic density of AXCs. The remaining logarithms of sums in the formula of the logarithmic density are typically numerically trivial, as all summands are bounded to lie in $[0,1]$. More importantly, the nested (exp-ln)-trick allows one to compute both (possibly high-order) derivatives $\psi^{(k)}$ and $\mathrm{D}_{B} \ell$ in logarithmic scale (see $b_{k}^{\psi, \ell}(\boldsymbol{u})$ and $a_{\pi}^{\psi, \ell, k}(\boldsymbol{u})$, respectively); the non-logarithmic values are never used. This is numerically an important result as the logarithmic terms can typically be implemented efficiently themselves; for $\ln \left\{(-1)^{k} \psi^{(k)}\right\}$ for well known Archimedean families see, e.g., [20, 21] or the R package copula of [19].

\section{References}

[1] S. Aulbach, M. Falk, M. Zott, The space of $d$-norms revisited, Extremes 18 (2015) 85-97.

[2] J. Beirlant, Y. Goegebeur, J. Segers, J. Teugels, Statistics of Extremes: Theory and Applications, Wiley, New York, 2004.

[3] L. Belzile, J.L. Wadsworth, P.J. Northrop, S.D. Grimshaw, R. Huser, mev: Multivariate Extreme Value Distributions, 2017. $\mathrm{R}$ package version 1.10 .

[4] S.N. Bernstein, Sur les fonctions absolument monotones, Acta Mathematica 52 (1928) 1-66.

[5] P. Capéraà, A.-L. Fougères, C. Genest, Bivariate distributions with given extreme value distributions, J. Multivariate Anal. 72 (2000) 30-49. 
[6] S. Castruccio, R. Huser, M.G. Genton, High-order composite likelihood inference for max-stable distributions and processes, J. Comput. Graph. Statist. 25 (2016) 1212-1229.

[7] A. Charpentier, A.-L. Fougères, C. Genest, J.G. Nešlehová, Multivariate Archimax copulas, J. Multivariate Anal. 126 (2014) 118-136.

[8] L. de Haan, A spectral representation for max-stable processes, Ann. Probab. 12 (1984) 1194-1204.

[9] A.B. Dieker, T. Mikosch, Exact simulation of Brown-Resnick random fields at a finite number of locations, Extremes 18 (2015) 301-414.

[10] C. Dombry, S. Engelke, M. Oesting, Exact simulation of max-stable processes, Biometrika 103 (2016) 303-317.

[11] G. Doyon, On Densities of Extreme Value Copulas, Master's thesis, ETH Zürich, Zürich, Switzerlanld, 2013.

[12] W. Feller, An Introduction to Probability Theory and Its Applications, vol. 2, 2nd edition, Wiley, New York, 1971.

[13] C. Genest, L.-P. Rivest, A characterization of Gumbel's family of extreme value distributions, Statist. Probab. Lett. 8 (1989) 207-211.

[14] M. Hardy, Combinatorics of partial derivatives, Electron. J. Combinatorics, 13 (2006) \#R1.

[15] M. Hofert, Sampling Nested Archimedean Copulas with Applications to CDO Pricing, Südwestdeutscher Verlag für Hochschulschriften AG \& Co. KG, 2010.

[16] M. Hofert, Efficiently sampling nested Archimedean copulas, Comput. Statist. Data Anal. 55 (2011) 57-70.

[17] M. Hofert, A stochastic representation and sampling algorithm for nested Archimedean copulas, J. Stat. Comput. Simul. $82(2012) 1239-1255$.

[18] M. Hofert, M. Scherer, CDO pricing with nested Archimedean copulas, Quantitative Finance 11 (2011) 775-787.

[19] M. Hofert, I. Kojadinovic, M. Mächler, J. Yan, copula: Multivariate Dependence with Copulas, 2005. R Package Version 0.999-19.

[20] M. Hofert, M. Mächler, A.J. McNeil, Likelihood inference for Archimedean copulas in high dimensions under known margins, J. Multivariate Anal. 110 (2012) 133-150.

[21] M. Hofert, M. Mächler, A.J. McNeil, Archimedean copulas in high dimensions: Estimators and numerical challenges motivated by financial applications, J. Soc. Franc. Statist. 154 (2013) 25-63.

[22] R. Huser, A.C. Davison, Composite likelihood estimation for the Brown-Resnick process, Biometrika 100 (2013) 1-9.

[23] J. Hüsler, R.-D. Reiß, Maxima of normal random vectors: Between independence and complete dependence, Statist. Probab. Lett. 7 (1989) 283-286.

[24] P. Jaworski, F. Durante, W.K. Härdle, T. Rychlik, editors. Copula Theory and Its Applications, Lecture Notes in Statistics - Proceedings, Springer, 2010.

[25] H. Joe, Multivariate Models and Dependence Concepts, Chapman \& Hall/CRC, London, 1997.

[26] Z. Kabluchko, M. Schlather, L. de Haan, Stationary max-stable fields associated to negative definite functions, Ann. Probab. (2009) 2042-2065.

27] D. Lee, H. Joe, Multivariate extreme value copulas with factor and tree dependence structures, Extremes 21 (2018) 147-176.

[28] A.W. Marshall, I. Olkin, Families of multivariate distributions, J. Amer. Statist. Assoc. 83 (1988) 834-841.

[29] D. McFadden, Modeling the choice of residential location, In: A. Karlqvist, F. Snickars, J. Weibull, editors, Spatial Interaction Theory and Planning Models, pp. 75-96, Elsevier North Holland, 1978.

[30] A.J. McNeil, Sampling nested Archimedean copulas, J. Stat. Comput. Simul. 78 (2008) 567-581.

[31] A.J. McNeil, J. Nešlehová, Multivariate Archimedean copulas, $d$-monotone functions and $\ell_{1}$-norm symmetric distributions, Ann. Statist. 37 (2009) 3059-3097.

[32] A.J. McNeil, R. Frey, P. Embrechts, Quantitative Risk Management: Concepts, Techniques, Tools, 2nd edition, Princeton University Press, Princeton, NJ, 2015.

[33] R. Mesiar, V. Jágr, $d$-dimensional dependence functions and Archimax copulas, Fuzzy Sets Syst. 228 (2013) 78-87.

[34] R.B. Nelsen, An Introduction to Copulas, 2nd edition, Springer, New York, 2006.

[35] A.K. Nikoloulopoulos, H. Joe, H. Li, Extreme value properties of multivariate $t$ copulas, Extremes 12 (2009) $129-148$.

[36] J.P. Nolan, Stable Distributions - Models for Heavy Tailed Data, Birkhäuser, Boston, 2017.

[37] T. Opitz, Extremal $t$ processes: Elliptical domain of attraction and a spectral representation, J. Multivariate Anal. 122 (2013) 409-413.

[38] M.D. Penrose, Semi-min-stable processes, Ann. Probab. 20 (1992)1450-1463.

[39] P. Ressel, Homogeneous distributions - and a spectral representation of classical mean values and stable tail dependence functions, J. Multivariate Anal. 117 (2013) 246-256.

[40] M. Schlather, Models for stationary max-stable random fields, Extremes 5 (2002) 33-44.

[41] A.G. Stephenson, Simulating multivariate extreme value distributions of logistic type, Extremes 6 (2003) 49-59.

[42] J.A. Tawn, Modelling multivariate extreme value distributions, 77 (1990) 245-253.

[43] S. Vettori, R. Huser, M.G. Genton, Bayesian clustering and dimension reduction in multivariate extremes, 2017. submitted. 